\title{
Piecewise constant averaging methods allow for fast and accurate hierarchical Bayesian estimation of drift diffusion models with time-varying evidence accumulation rates.
}

\author{
Gaia Lombardi $^{1} \quad$ | Todd A. Hare ${ }^{1}$
}

${ }^{1}$ Department of Economics, University of Zurich, Zurich, 8006, Switzerland

\section{Correspondence}

Gaia Lombardi, Department of Economics, University of Zurich, Zurich, 8006,

Switzerland

Email: gaia.lombardi@econ.uzh.ch

\section{Funding information}

The work in this study benefited from the support of Swiss National Science Foundation (SNSF) grants $320030 \_143443$ and 32003B _166566 and EU FP7 grant 607310 (NUDGE-IT).
Computational models of neurons and behavior are key tools in the feedback cycle between theory development, experimentation, and data analysis. The drift diffusion model (DDM) and other types of sequential sampling models have proven to be useful ways of quantifying and characterizing individual differences and the effects of experimental manipulations on various types of behavior in humans and other animals. To date, partially for the sake of simplicity, most modeling studies have assumed a constant rate of evidence accumulation when fitting the data and testing theoretical predictions. Nevertheless, there are a growing number of theories and empirical data that suggest that evidence accumulation rates may vary within the time course of a decision because of changes in attention, arousal, goals, or other factors. Fitting a DDM with a time-varying evidence accumulation rate can be much more computationally demanding and time consuming than fitting the standard DDM if there is no analytical solution for the timevarying DDM. Here, we demonstrate how to mathemati- 
cally reformulate an influential subset of time-varying DDMs into standard DDMs with constant drift rates. This simple yet powerful reformulation allows time-varying DDMs with piecewise-constant drift rates to be easily and rapidly estimated within existing hierarchical Bayesian frameworks. We show detailed examples of this process using data from both computer simulations and humans in two separate empirical studies. Our results demonstrate that the method can quickly and accurately recover parameters from simulations and fit hierarchical Bayesian models to real data.

\section{KEYWORDS}

Hierarchical Bayesian methods, time-varying diffusion models, decision-making, sequential sampling

\section{1 | INTRODUCTION}

Noisy accumulation of evidence to a decision bound has proven to be an important and influential concept within models of value-based and perceptual decisions in the social and biological sciences. This process is at the core of widely used sequential sampling models such as the drift diffusion model (DDM, [25, 26, 27, 37]), decision field theory (MDFT, [5, 28, 12]), the leaky competing accumulators model (LCA, [32]), the association and accumulation model (AAM, [2]), the linear ballistic accumulator (LBA, [4]), and various others.

To date, most attempts to fit this class of model to empirical data have assumed a constant evidence accumulation or drift rate throughout the choice process (e.g. the standard DDM or the LCA). The computational complexity and time required to fit models that relax the assumption of a constant evidence accumulation rate have been key reasons for maintaining this assumption in most empirical applications. The rise in popularity of hierarchical Bayesian estimation methodologies [33, 39, 34] may have further dissuaded researchers from using time-varying drift rate models because Bayesian estimation using popular Markov-chain Monte Carlo (MCMC) methods typically requires an analytical solution for equations within the model in order to generate the posterior distribution within a reasonable time frame.

Bayesian data analytic methods have at least two benefits for the decision sciences. First, Bayesian methods allow inference on the entire distribution of the parameters rather than just the most likely estimate as in methodologies based on maximum likelihood (MLE). These full distributions of parameters are useful because they provide a measure of the uncertainty in the parameters' values. This explicit uncertainty is useful in terms of both assessing the quality of model fits to data, and the interpretation of the parameters themselves. Second, hierarchical structures are efficiently embedded in the Bayesian framework in a readily interpreted manner. Standard modelling methodologies have to assume either complete independence in the data structure (i.e., as in single-subject MLE) or complete pooling (i.e., fitting averaged data and implicitly assuming that all participants are identical) [19, 31, 30]. In contrast, hierarchical Bayesian modelling implements a compromise between the two strategies allowing group and subject parameters to be estimated simultaneously at different hierarchical levels, thereby providing robust estimates of a model's free parameters without ignoring or over-weighting individual differences. In other words, Bayesian models can allow for 
the more complete theoretical explanation of data from a single task, letting different people use different cognitive processes, or letting the same people use different processes at different times. However, as mentioned above, efficiently generating the posterior distribution of the parameters with hierarchical Bayesian MCMC sampling methods is greatly facilitated when there is an analytical solution for equations within the model framework. Analytical solutions are not always possible to mathematically derive for models with time-varying evidence accumulation (i.e. drift) rates. Although there are means of approximating the analytical solution for some types of time-varying drift rate DDMs [29], it is often theoretically and computationally challenging to estimate and implement such formulations.

Despite the added complexity of their estimation, time-varying drift rate models are important because there are many types of decision strategies and mechanisms that cannot be modeled with a fixed drift rate. Examples of sequential sampling models in the existing literature that have a time-varying drift rate are, the attentional drift diffusion model (aDDM), [15, 16, 6], the dual-stage two-phase (DSTP) model [13], the piecewise linear ballistic accumulator (pLBA), [11, 10] and the relative starting-time drift diffusion model (stDDM), [1, 18]. All of the models listed above share the fact that they do not have completely time-dependent drift rates in the sense of a linear or quadratic relation to time. Instead the drift rate in all of these models is time-constant in fixed or alternating intervals, i.e. it is piecewise time-constant. Critically, if the intervals are known, then diffusion models with piecewise time-constant drift rates ( $p c D D M)$ can be re-written as standard drift diffusion models (DDM) for which the drift rate is constant in time using simple algebra. Here, we show how a simple, yet powerful, piecewise constant averaging method allows for the use of existing hierarchical Bayesian estimation tools that were developed for the standard DDM to fit the time-varying drift rates of any DDM for which we can compute the average drift rate over an observable period of time. The advantage of using well-establish standard DDM methodologies can be found in the faster speed of estimation and in the simplicity of implementation when compared to other methods such as the martingales method [29] or the probability density approximation (PDA) method [30, 9, 10].

In this paper, we illustrate how to transform a pcDDM into a standard DDM with a fully time-constant drift rate. We will show that a piecewise time-constant drift rate can be expressed as a constant drift that is a function of the first passage time - i.e reaction time - and the times for each constant interval. We also walk through two concrete examples of how to apply this methodology. We derive the piecewise time-constant drift for the aDDM and for the stDDM, and implement these drifts in a hierarchical Bayesian DDM estimation framework implemented in R and JAGS. We then show the recovery fitting analysis of the two models to demonstrate that 1) we accurately recover the timevarying parameters through this reparameterization of the standard DDM, and 2) that the estimates of the other DDM parameters are not adversely affected by the inclusion of these extra parameters for the drift rate. Lastly, we report the parameter estimates from two piecewise-constant drift diffusion models and show goodness of fit measures for the experimental reaction time and choice distributions from two experiments in humans.

\section{2 | RESULTS}

We first introduce the formulation of the standard drift diffusion model (DDM) in its continuous version as an stochastic differential equation (SDE) and a general piecewise time-constant drift diffusion model (pcDDM) in a similar formulation. We will then demonstrate in the next sections that the drift rate of the pcDDM can be rewritten as a constant drift rate as in the DDM under the conditions that the stopping time and the intervals are explicitly specified. 


\subsection{Standard drift diffusion model (DDM)}

A standard drift diffusion model can be expressed as a stochastic differential equation (SDE) that makes use of stochastic accumulation in a continuous time framework. The SDE can be written as

$$
\begin{aligned}
\mathrm{d} x(t) & =\mu \mathrm{d} t+\sigma \mathrm{d} W(t) \quad x(0)=x_{0}, \\
\tau & =\inf \{t>0 \mid x(t) \notin(-B, B)\}
\end{aligned}
$$

where $x(t)$ is the evidence accumulated at time $t, \tau$ the first passage time (or reaction time) when the evidence crosses a certain threshold $\mathrm{B}, \mu$ is the drift rate or speed of the accumulation process, $\sigma$ is the diffusion rate or standard deviation of the Brownian motion, $x_{0}$ is the initial bias and $\epsilon_{t} \sim \mathrm{N}\left(0, \sigma^{2}\right)$.

In equation 1, the evidence $x(t)$ evolves in time according to a biased random walk with Gaussian increments, i.e. $\mathrm{d} x(t) \sim \mathrm{N}\left(\mu \mathrm{d} t, \sigma^{2} \mathrm{~d} t\right)$, and the analytical solution for the distribution of the reaction time $\tau$ can be mathematically derived when the drift rate $\mu$ and diffusion rate $\sigma$ are constant in time [20].

\section{2 | General piecewise constant DDM (pcDDM)}

As noted in the introduction section of the paper, some of the most prominent time-varying DDMs found in literature, e.g. the aDDM, do not have fully time-dependent drift rates, but rather have drift rates that are constant over discrete intervals. The continuous SDE of such a model (pcDDM) can be written as follows.

$$
\begin{aligned}
& \mathrm{d} x(t)=\mu(t) \mathrm{d} t+\sigma \mathrm{d} W(t) \quad x(0)=x_{0}, \\
& \mu(t)=\mu_{i}, \quad \text { for } t_{i} \leq t<t_{i+1} \\
& \tau=\inf \{t>0 \mid x(t) \notin(-B, B)\}
\end{aligned}
$$

We can now see that the drift rate can be rewritten as follows when the intervals in which it is constant and the stopping time are explicitly specified.

$$
\begin{aligned}
\mu(t) & =\sum_{i=0}^{n-1} \mu_{i} \mathbb{1}\left[t_{i}, t_{i+1}\right)(1) \\
\int_{0}^{\tau} \mu(t) \mathrm{dt} & =\int_{0}^{\tau} \sum_{i=0}^{n-1} \mu_{i} \mathbb{1}\left[t_{i}, t_{i+1}\right)(1) \mathrm{dt} \\
& =\sum_{i=0}^{n-1} \int_{0}^{\tau} \mu_{i} \mathbb{1}\left[t_{i}, t_{i+1}\right)(1) \mathrm{dt} \\
& =\sum_{i=0}^{n-1} \int_{t_{i}}^{t_{i+1}} \mu_{i} \mathrm{dt} \\
& =\tau\left(\sum_{i=0}^{n-1} \int_{t_{i}}^{t_{i+1}} \mu_{i} \mathrm{dt}\right) \frac{1}{\tau} \\
& =\int_{0}^{\tau} \frac{\mathrm{d}}{\mathrm{d} \tau}\left[\tau\left(\sum_{i=0}^{n-1} \int_{t_{i}}^{t_{i+1}} \mu_{i} \mathrm{dt}\right) \frac{1}{\tau}\right] \mathrm{dt} \\
& =\int_{0}^{\tau}\left(\frac{1}{\tau} \sum_{i=0}^{n-1} \int_{t_{i}}^{t_{i+1}} \mu_{i} \mathrm{dt}\right) \mathrm{dt}
\end{aligned}
$$


where $\mathrm{n}$ is the number of intervals, $t_{0}=0$ and $t_{n}=\tau$

$$
\begin{aligned}
\mu(t) & =\frac{1}{\tau} \sum_{i=0}^{n-1} \int_{t_{i}}^{t_{i+1}} \mu_{i} \mathrm{dt} \\
& =\frac{1}{\tau} \sum_{i=0}^{n-1} \mu_{i}\left(t_{i+1}-t_{i}\right)
\end{aligned}
$$

Thus, if the first passage time and the intervals are known, the time-continuous form of the pcDDM can be written as

$$
\begin{aligned}
& \mathrm{d} x(t)=\bar{\mu} \mathrm{d} t+\sigma \mathrm{d} W(t) \quad x(0)=x_{0}, \\
& \bar{\mu}=\frac{1}{\tau} \sum_{i=0}^{n-1} \mu_{i}\left(t_{i+1}-t_{i}\right), \\
& \tau=\inf \{t>0 \mid x(t) \notin(-B, B)\}
\end{aligned}
$$

where $\bar{\mu}$ is a function of first passage time $\tau$, the number of intervals $n$ and their duration $t_{i+1}-t_{i}$.

We, thus, demonstrated that the drift rate of a pcDDM when the intervals in which it is constant and the stopping time are explicitly known is equivalent to a constant drift rate that is the average in time of the original formulation. Figure 1 provides an illustration of two simulations of the aDDM, the model displayed by the black line has been simulated with the standard drift rate as in equation 6 and the red line shows a simulation of the aDDM with the average drift rate as derived above and in equation 7. Converting a piecewise-constant DDM into one with a constant slope allows us to derive the distribution of response times analytically rather than relying on numerical simulations, which in turn means that we can fit Bayesian models using MCMC procedures in a reasonably small amount of time.

In the next section, we will show the drift rate formula $\bar{\mu}$ for two specific example pcDDMs, the aDDM and the stDDM. 
(a)

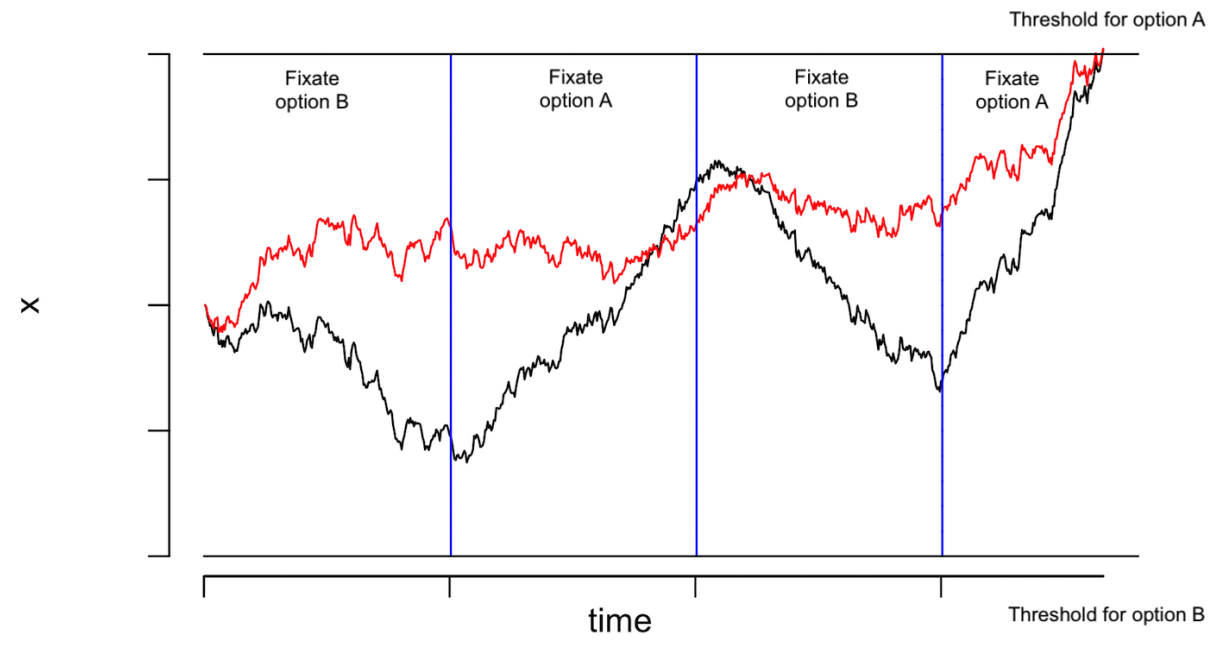

(b)

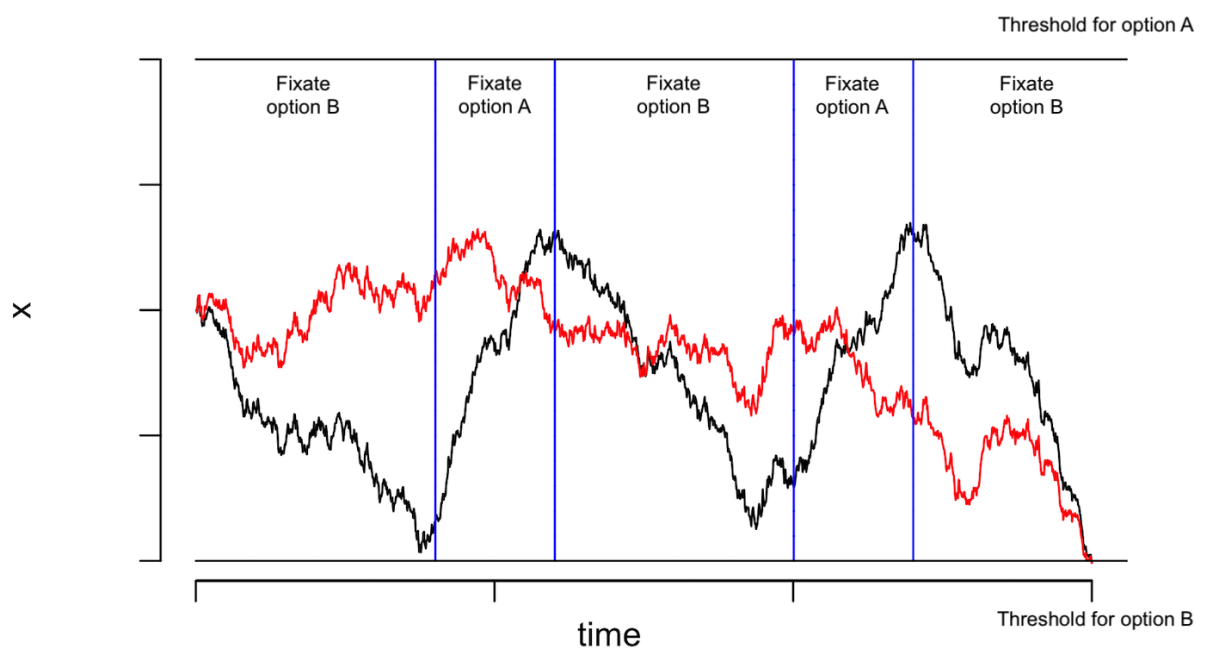

FIGURE 1 Evidence accumulation traces with time-varying drift rates versus a drift rate equal to the time-weighted average of the drift rates within each interval. The two plots show simulations of the attention drift diffusion model (aDDM) with standard fixation-location-dependent drift rate as in equation 6 (black line), and with a drift rate equal to the weighted average of the rates across all intervals as in equation 7 (red line). The blue vertical lines denote a change between intervals within which the evidence accumulation rate is constant. In the case of the aDDM, the end of an interval is marked by a change in fixation from option B to the option $A$. a) In this example, both options receive the same amount of fixation pattern. b) In this example, one option $B$ is fixated longer than the option $A$, resulting in a change in the outcome and the weighted average of the drift rate compared to a). The fact that we know 1) the time at which the evidence accumulation process reaches a threshold (i.e. the response time), and 2) the duration of each interval within which the drift rate is constant (i.e. fixation locations and durations) for each trial means that one can compute the weighted average of the piecewise constant drift rates for each individual trial in a data set. Using these trial-specific weighted averages, one can fit a piecewise constant DDM as if it had entirely constant evidence accumulation using the closed form solution for constant drift rates instead of numerical simulation. Note that any parameter that influences the piecewise constant drift rates will also influence their weight average and thus parameters scaling the relative values of the two options or fixation-dependent discounting can be optimized across trials. 


\section{$2.3 \mid a D D M$}

The aDDM model [15] is an example of a modified version of a standard DDM for which the drift rate varies in time. The basic idea of the model is that the drift rate changes every time the decision maker visually fixates one option or another. The continuous aDDM formula is the following.

$$
\begin{aligned}
& \mathrm{d} x(t)=\mu(t) \mathrm{d} t+\sigma \mathrm{d} W(t) \quad x(0)=x_{0}, \\
& \mu(t)= \begin{cases}\delta\left(V_{A}-\theta V_{B}\right) & \text { if option A is attended at time } t \\
\delta\left(\theta V_{A}-V_{B}\right) & \text { if option B is attended at time } t\end{cases} \\
& \tau=\inf \{t>0 \mid x(t) \notin(-B, B)\} .
\end{aligned}
$$

where $\theta$ is the discount factor that penalises the value of the unattended option, $\delta$ is the drift scaling parameter and $V_{A}$ and $V_{B}$ are the values of option $A$ and $\mathrm{B}$, respectively. Thus, knowing the total fixation time for option $\mathrm{A}$ and option $\mathrm{B}$, we can rewrite the drift rate for the aDDM as we did in equation 5 for the a general pcDDM.

$$
\begin{aligned}
& \mathrm{d} x(t)=\bar{\mu} \mathrm{d} t+\sigma \mathrm{d} W(t) \quad x(0)=x_{0}, \\
& \bar{\mu}=\frac{\tau_{A}}{\tau} \delta\left(V_{A}-\theta V_{B}\right)+\frac{\tau_{B}}{\tau} \delta\left(\theta V_{A}-V_{B}\right) \\
& \tau=\inf \{t>0 \mid x(t) \notin(-B, B)\}
\end{aligned}
$$

where $\tau_{A}$ and $\tau_{B}$ are the total fixation times to option $A$ and option $B$, respectively.

\section{4 stDDM}

The relative-starting-time DDM (stDDM) [18] is another example of a piecewise constant DDM for multi-attribute choices. This formulation of the DDM includes a relative-starting-time parameter that allows for one attribute to begin being considered before another. Other than that additional parameter, the model is a basic drift diffusion model, in which the drift rate depends on the weighted value differences of two attributes. This model can be used for different types of options that have two or more attributes, for example lotteries with money and probabilities or food items with healthiness and palatability attributes. The key idea of the model is that one of the two attributes might have some delay in entering the accumulation process relative to the other. For instance, a decision maker that has to choose between a salad and a chocolate cake might immediately think about the delicious taste of the cake compared to the salad, and only after half a second start to consider the fact that the chocolate cake would be a far less healthy option.

The continuous formulation of the stDDM can be written as follows.

$$
\begin{aligned}
& \mathrm{d} x(t)=\bar{\mu} \mathrm{d} t+\sigma \mathrm{d} W(t) \quad x(0)=x_{0}, \\
& \mu(t)= \begin{cases}w_{T} \mathrm{VD}_{T} & \text { if } s>0 \wedge 0<t<s \\
w_{H} \mathrm{VD}_{H} & \text { if } s<0 \wedge 0<t<|s| \\
w_{H} \mathrm{VD}_{H}+w_{T} \mathrm{VD}_{T} & \text { if } t>|s|\end{cases} \\
& \tau=\inf \{t>0 \mid x(t) \notin(-B, B)\}
\end{aligned}
$$

where $w_{T}$ is the weight given attribute $T, w_{H}$ the weight attribute $\mathrm{H}$ (T and $\mathrm{H}$ being any two attributes), $\mathrm{VD}_{T}$ and $\mathrm{VD}_{H}$ are the value differences in attributes $\mathrm{T}$ and $\mathrm{H}$ respectively, $s$ is time at which attribute $\mathrm{H}$ comes into the accumulation 
process $-s>0$ means T-related evidence is accumulated from the beginning and evidence from attribute $\mathrm{H}$ comes into consideration at time $s$, while $s<0$ means that attribute $\mathrm{H}$ influences the accumulation process from the beginning and evidence from attribute $\mathrm{T}$ starts to come in at time $|s|$.

Once again, knowing the stopping time $\tau$ and explicitly specifying a relative starting time $s$, we can determine the average rate as follows

$$
\begin{aligned}
\mathrm{d} x(t) & =\bar{\mu} \mathrm{d} t+\sigma \mathrm{d} W(t) \\
\bar{\mu} & = \begin{cases}\frac{s}{\tau} W_{T} \mathrm{VD}_{T}+\frac{\tau-s}{\tau}\left(w_{H} \mathrm{VD}_{H}+w_{T} \mathrm{VD}_{T}\right) & \text { if } s>0 \wedge s<\tau \\
\frac{|s|}{\tau} w_{H} \mathrm{VD}_{H}+\frac{\tau-|s|}{\tau}\left(w_{H} \mathrm{VD}_{H}+w_{T} \mathrm{VD}_{T}\right) & \text { if } s<0 \wedge|s|<\tau \\
w_{T} \mathrm{VD}_{T} & \text { if } s>0 \wedge s>\tau \\
w_{H} \mathrm{VD}_{H} & \text { if } s<0 \wedge|s|>\tau\end{cases} \\
\tau & =\inf \{t>0 \mid x(t) \notin(-B, B)\}
\end{aligned}
$$

In summary, we have shown that both the aDDM and the stDDM can be written as a standard DDM for which the drift rate is a function of the reaction time and the duration of the constant intervals (i.e. relative fixation time advantage or relative starting time advantage) in a given choice or trial. The advantage of this formulation is that we have an analytical solution for the pcDDMs that is identical to the analytical solution of the DDM [20]. Thus, a hierarchical Bayesian approach can now be easily applied to this type of model. Here, we implement the piecewise constant averaging method described above within a hierarchical Bayesian estimation framework giving us the hierarchical Bayesian attention drift diffusion model ( $\mathrm{HaDDM})$ and the hierarchical Bayesian relative-start-time DDM (HstDDM). We coded the HaDDM and the HstDDM in JAGS using equations 7 and 9 above, and performed recovery fitting analysis in R (see the Methods section for details and https://github.com/galombardi/ for the full code). All the simulations were performed with the Euler method as standard practice in the field.

Below, we report and discuss tests of the parameter recovery accuracy for both models.

\section{5 | Recovery fitting analysis}

To further assess and demonstrate the validity of this method in practical terms, we performed a parameter recovery analysis for the HaDDM and the HstDDM. The aim of this recovery fitting analysis was twofold. Firstly, it shows the ability of the method to recover different parameter combinations at the individual and the group level and, in particular, that the mean of the posterior distributions from the recovery of each parameter is informative of the real generating values. Secondly, it shows that this fitting method is able to recover different parameters for different individuals from the same population. We performed parameter recovery with two types of participant samples, A) samples of participants with homogeneous parameters (i.e. identical for all individuals) so that we could test several different known combinations of parameters at the group level, and B) a participant sample with a heterogeneous distribution of parameters across individuals so that we could determine recovery accuracy at the individual level. In the homogeneous sample tests, for both the HaDDM and the HstDDM, the sets of parameters we tested varied around the best fitting parameter estimates from past fits to empirical data sets. For the heterogeneous samples, each of the parameters were randomly drawn from Gaussian distributions with a fixed mean and standard deviation. All simulations for both pcDDMs were performed with the number of subjects, trials, and value differences taken from existing empirical data sets (see Methods section for details). Note, that each subject for our simulations differs from the others in value difference trials and, concerning the aDDM, in fixation patterns (i.e., fixation duration and 
proportion). Although the piece-wise constant averaging method can be used to fit time-varying DDMs to existing data, one cannot use it to simulate new data. This is because the method requires us to know the response times and constant interval durations. Therefore, both models were simulated with the Euler method approximation, also known as the discrete bounded accumulation series as follows. The series for the aDDM is

$$
\begin{aligned}
x_{t_{i}} & =x_{t_{i-1}}+\mu_{t_{i}}\left(t_{i}-t_{i-1}\right)+\epsilon_{t_{i}} \sqrt{\left(t_{i}-t_{i-1}\right)} \\
\mu_{t_{i}} & = \begin{cases}\delta\left(V_{A}-\theta V_{B}\right) & \text { if option A is attended at time } t_{i} \\
\delta\left(\theta V_{A}-V_{B}\right) & \text { if option B is attended at time } t_{i}\end{cases} \\
\tau & =t_{N}=\min \left\{t_{i}, \forall i \in \mathbb{N}|| x_{t_{i}} \mid>B\right\} .
\end{aligned}
$$

The stDDM discrete bounded accumulation series is

$$
\begin{aligned}
x_{t_{i}} & =x_{t_{i-1}}+\mu_{t_{i}}\left(t_{i}-t_{i-1}\right)+\epsilon_{t_{i}} \sqrt{\left(t_{i}-t_{i-1}\right)} \\
\tau & =t_{N}=\min \left\{t_{i}, \forall i \in \mathbb{N}|| x_{t_{i}} \mid>B\right\} \\
\mu_{t_{i}} & = \begin{cases}w_{T} \mathrm{VD}_{T} & \text { if } s>0 \wedge 0<i<s \\
w_{H} \mathrm{VD}_{H} & \text { if } s<0 \wedge 0<i<|s| \\
w_{H} \mathrm{VD}_{H}+w_{T} \mathrm{VD}_{T} & \text { if } i>|s|\end{cases}
\end{aligned}
$$

For all samples and versions of the diffusion model, we fitted a hierarchical Bayesian version of the model. Hierarchical Bayesian estimation procedures estimate the posterior distributions of each parameter at the individual and group levels using a specified hierarchical relationship (i.e. assuming that individual parameters are somewhat similar and drawn from a common distribution). This approach is more data efficient than estimating each individual separately because it allows for data sharing across individuals. The degree of assumed similarity between individuals, and thus regularization of the individual parameters toward the group mean, is determined by the priors used for the group-level parameters. Such methods allow one to make inference about the population distributions of the parameters, while explicitly accounting for variability due to individual differences.

The next sections show that the piecewise constant averaging method was able to accurately estimate the parameters for both the HaDDM and the HstDDM.

\subsection{1 | HaDDM}

We first tested parameter recovery accuracy for the HaDDM using a sets of agents ( $N=30$ agents per set) that all made choices based on the same underlying model parameters, but each agent had different fixation patterns and subjective values for the lotteries. In all simulations, we used the fixation and subjective value data from an existing data set that is described in section 2.6 and in the Methods section. We simulated the bounded accumulation series of the aDDM for several different parameter combinations. Simulating multiple agents with the same aDDM parameters, but different fixation patterns and trial types (trial type is determined by the subjective value of the lotteries) allowed us to test the recovery accuracy and any potential bias in the fitting method. The parameters' values were based on the range of parameters estimated for choices from real human subjects. The attention discount factor $\theta$ was drawn from the set $\{0.2,0.4,0.6,0.8\}$, the drift scaling parameter $\delta$ from $\{6,10,14\}$ and the standard deviation of the noise $\sigma$ from $\{0.3,0.45,0.6\}$, and then we simulated choices from samples having all the possible parameter combinations. 


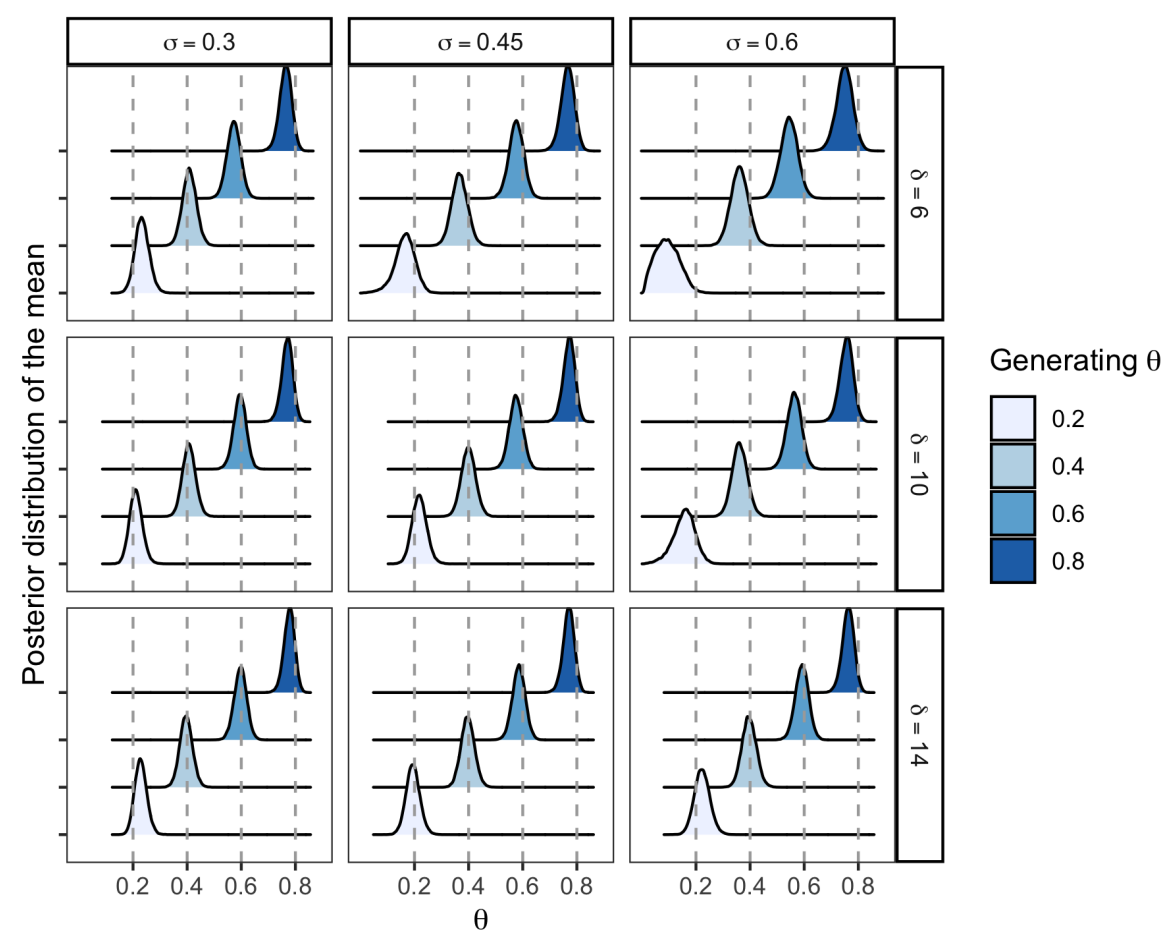

FIGURE 2 Hierarchical group level posterior probability distributions of the mean $\theta$ parameters from the HaDDM fits of simulations varying the $\delta$ and the $\sigma$ parameters. Each posterior distribution is based on a hierarchical Bayesian fit to 30 independent simulations. The dashed grey lines indicate the input generating parameters for the simulations.

Lastly, we fit the HaDDM using the piecewise constant averaging method to all 30 agents in each set to estimate both individual and group-average parameters, and evaluated the recovered posterior distributions of the parameters for all of the various combinations.

In figure 2, we show the posterior distribution of the group-level mean of the discount parameter $\theta$ varying the drift scaling parameter $\delta$ and the standard deviation of the noise $\sigma$. Notably, the generating $\theta$ parameter was recovered well in all of the different simulations with a correlation between generating and recovered parameters of 0.95 and a mean squared error relative to the average value of $\theta(\mathrm{MSE} / \bar{\theta})$ of 0.015 . However, as expected, increasing the noise or decreasing the drift rate scaling parameter decreases the relative accuracy of the parameter recovery. This was also confirmed by an increase in correlation for higher values of the drift rate parameter and for lower values of the noise parameters $\left(\operatorname{corr}(\theta, \hat{\theta})_{\delta=6}=0.94, \operatorname{corr}(\theta, \hat{\theta})_{\delta=10}=0.95, \operatorname{corr}(\theta, \hat{\theta})_{\delta=14}=0.97, \operatorname{corr}(\theta, \hat{\theta})_{\sigma=0.3}=0.99\right.$, $\left.\operatorname{corr}(\theta, \hat{\theta})_{\sigma=0.6}=0.94\right)$.

We then confirmed that varying the attention discount factor $\theta$ does not influence the recovery accuracy of the other parameters. In Figures S1 and S2, we show the ability of the model to recover the generating $\delta$ and $\sigma$ parameters respectively, varying the other two parameters. The piecewise constant averaging method is able to accurately recover all the various parameters. The correlations and the MSE at the level of were $\operatorname{corr}(\delta, \hat{\delta})=0.99$, $\operatorname{corr}(\sigma, \hat{\sigma})=0.96, \frac{\operatorname{MSE}(\delta)}{\bar{\delta}}=0.067$, and $\frac{\operatorname{MSE}(\sigma)}{\bar{\sigma}}=0.003$, respectively.

However, it is worth noting that there is a systematic overestimation of the drift scaling parameter $\delta$ on average (see also the regression models at the individual level in Table S1). A plausible explanation for this overestimation is the fact that the discrete bounded accumulation series we used to simulate data is a numerical approximation of the continuous analytical solution of the model used to fit the parameters in the HaDDM. In other words, the bias we 
observe in the estimation may not be due to the piecewise constant averaging method itself, but rather is the result of an approximation error between the discrete bounded accumulation series we used to simulate the data and the analytical solution of the DDM specified as a continuous stochastic differential equation.

To test this hypothesis, we ran parameter recovery tests for the standard DDM using simulated choices and response times generated by either the discrete bounded accumulation series or the analytical solution of the continuous stochastic differential equation in 1 . We then estimated the best-fitting parameters for these simulated data sets using the same hierarchical Bayesian method that we used to fit the standard DDM to the initial empirical data to obtain the generating parameters for our simulations. We use the standard DDM to test this hypothesis because the piecewise constant averaging method requires us to know the duration of each interval and response time, and thus it cannot be used to generate choice outcomes and response times for simulated agents.

As predicted, we find that, when choices are simulated with the bounded accumulated series, the drift rate scaling parameter in a standard DDM has the same systematic overestimation that we see for the aDDM (and in the stDDM reported in the next section). Critically, when the choice response times are simulated with the continuous version of the DDM as the generating model, the bias is significantly reduced, Figure S3 and Table S4.

Thus, these results indicate that much of the small bias stems from simulating the diffusion models with the bounded accumulation series, which is a numerical approximation of the continuous time model. This small bias remains largely unchanged even when the step size in the numerical approximation is reduced from 1 to $0.01 \mathrm{~ms}$. Importantly, if the evidence accumulation process in humans or other animals operates in continuous time, then it will not be a source of bias. In any case, this bias is not problematic because it is a small, constant overestimation of the drift scaling parameter. That being the case, it does not interfere with our ability to distinguish differences between individuals or groups. Detecting such differences is generally the goal when fitting DDMs because the magnitudes of individual parameter estimates have little meaning on their own. We show in the next section that the overestimation of the drift scaling parameter is even smaller at the individual individual agents than it is at the hierarchical groupaverage level (see Figure 5).

Analyses of the differences between two known values of generating and recovered $\delta$ parameters showed that the piecewise constant averaging method is able to accurately distinguish between different generating $\delta$ parameters. Specifically, we calculated the posterior density of the difference between the drift scaling parameters, $\delta$, from recovery fits for two different simulations, and compared it with the difference in the generating parameters. Figure S4 shows that the recovered difference in $\delta$ estimates between simulations was highly accurate. We also computed a linear regression model on the difference between estimated $\delta$ parameters across different simulations and recov eries and found that there is no significant bias in the recovered differences (see Model (1) in Table S3). This is critical because in most cases, if not all, researchers will be fitting time-varying DDMs to compare across different individuals or experimental conditions. 
(a)

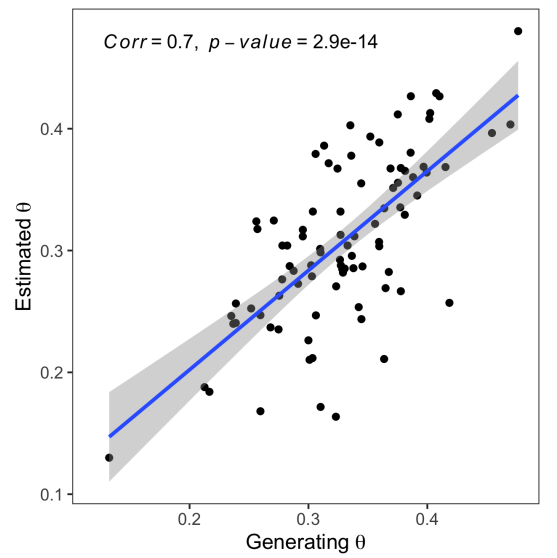

(b)

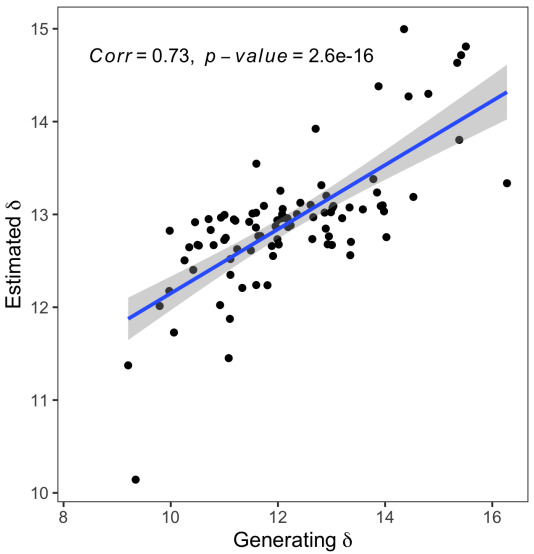

(c)

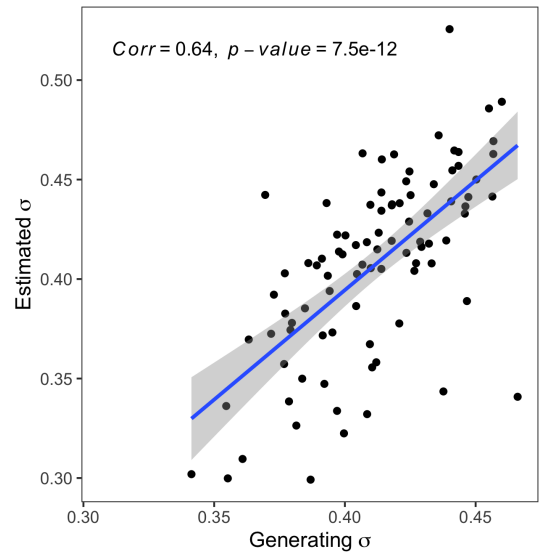

FIGURE 3 Correlation at the individual level between generating parameters and recovered parameters from the heterogeneous recovery fitting of the HaDDM. a) $\theta$ parameter. b) $\delta$ parameter. c) $\sigma$ parameter.

\section{| Fitting the HaDDM to groups of agents with heterogeneous model parameters}

It is also important to check whether our hierarchical Bayesian estimation method yields accurate estimates at the individual when fitting groups of heterogeneous individuals. Indeed, often it is the nature and magnitudes of differences between individuals in the sample that are the question of interest. Therefore, we simulated data using different generating parameters for each agent/subject in the group. The generating parameters were randomly drawn from the gaussian distributions shown in Figure S5a. We were able to accurately recover the parameters on the individual level. Figure 4, Figure 5 and Figure S6 show the recovery at the individual level for each subject for the $\theta$, the $\delta$ and the $\sigma$ parameters, respectively. 


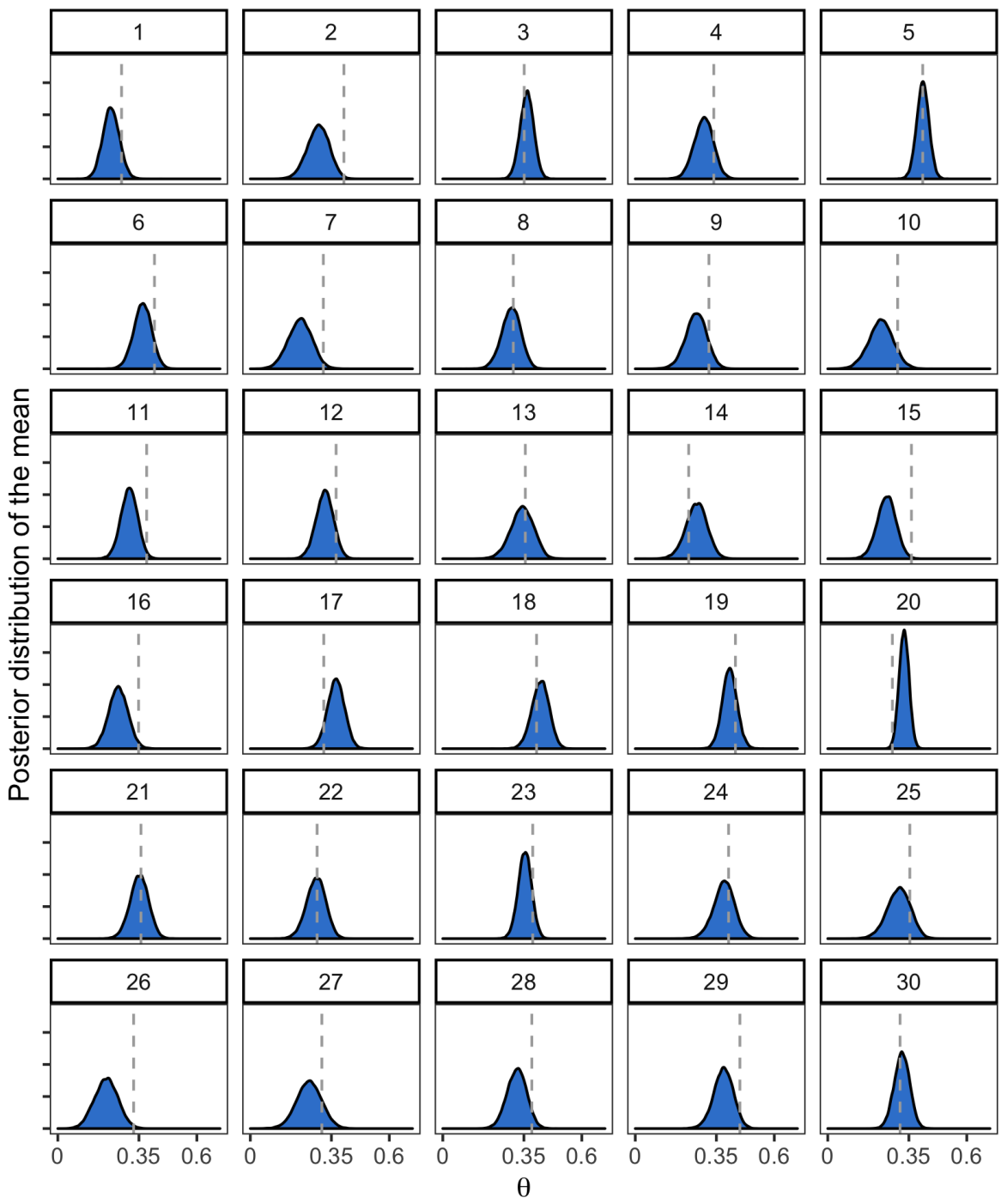

FIGURE 4 Individual level posterior probability distributions of the mean $\theta$ parameters from the HaDDM fits for each subject in the data set. The dashed grey lines indicate the input generating parameters used for the simulations.

\subsection{2 | HstDDM}

We repeated the same procedures described above for the aDDM to test parameter recovery from the stDDM. Thus, we simulated choice data from several combinations of parameters that were drawn from a range of plausible parameters based on fits to human behavior. The relative starting time parameter $s$ was taken from the set $\{-0.75,-0.5,-0.25,0,0.25,0.5,0.75\}$, and the standard deviation of the noise, $\sigma$, was drawn from $\{0.3,0.45,0.6\}$. The weighting parameters for different attributes are always defined relative to one another, therefore, we kept one fixed and varied the other. Specifically, $w_{t}$ was fixed to 2.2 and $w_{h}$ was taken from the set, $\{1.9,2.2,2.9\}$.

Figure 6 shows the recovered relative start time parameter $s$ for different values of $\sigma$ and $w_{h}$. The plot shows the posterior distribution of the hierarchical group level mean of the $s$ parameter across 45 simulated agents that all used the same stDDM parameters, but each faced a unique set of decision trials. These results demonstrate that a HstDDM based on our piecewise constant averaging method can accurately recover known relative starting time parameters. Figures S7, S8 and S9 show the posterior distributions of the other parameters. The correlation 


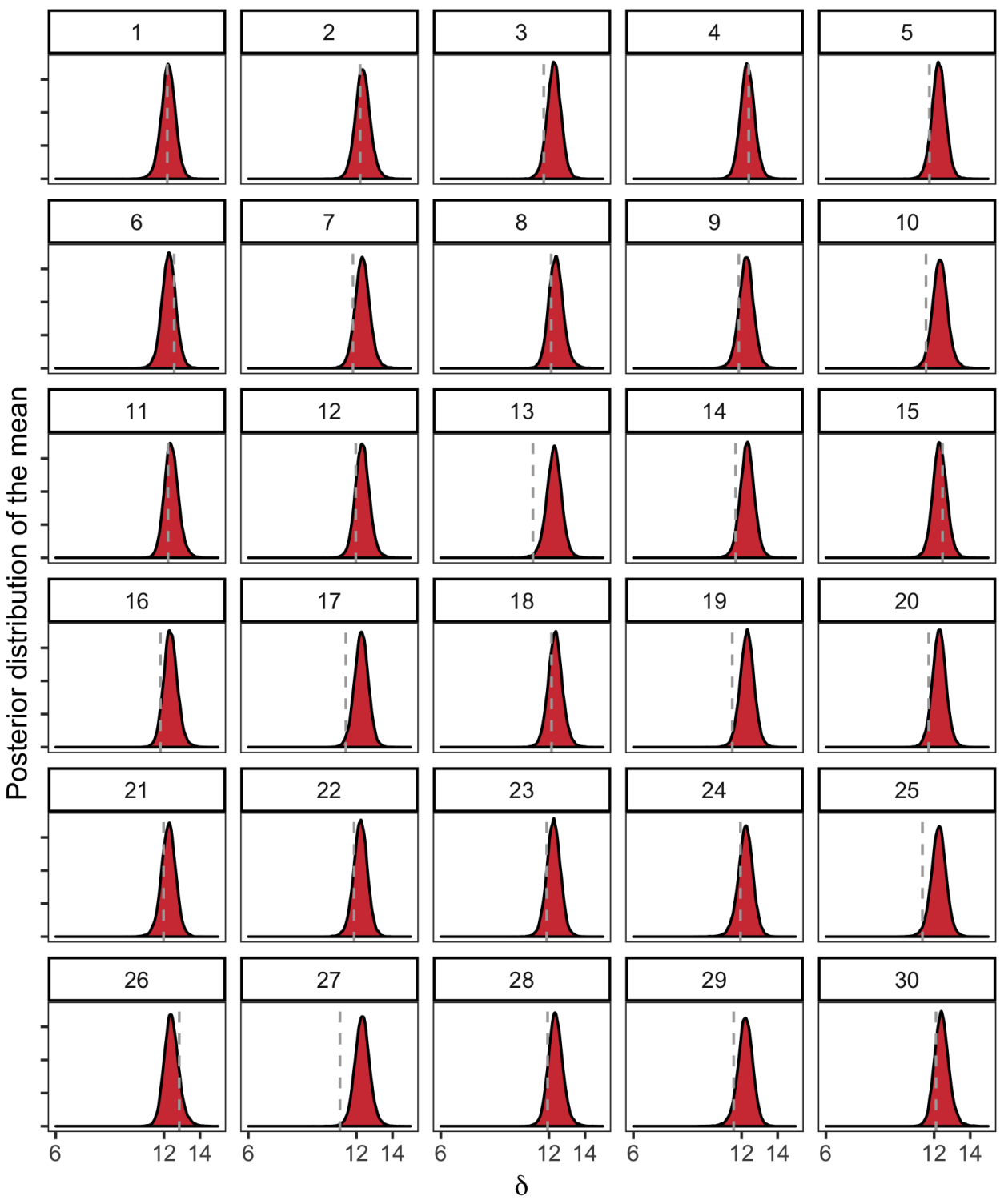

FIGURE 5 Individual level posterior probability distributions of the mean $\delta$ parameters from the HaDDM fits for each subject in the data set. The dashed grey lines indicate the input generating parameters used for the simulations.

and the MSE further confirm the ability of the method. $\operatorname{corr}(s, \hat{s})=0.99, \operatorname{corr}\left(w_{h}, \hat{w}_{h}\right)=0.98, \operatorname{corr}(\sigma, \hat{\sigma})=0.96$, $\operatorname{MSE}(s)=0.001, \frac{\operatorname{MSE}\left(w_{h}\right)}{\bar{w}_{h}}=0.013$, and $\frac{\operatorname{MSE}(\sigma)}{\bar{\sigma}}=0.006$.

As expected based on the recovery analysis for the HaDDM, there is a small overestimation of parameter values defining the drift scaling terms, i.e. the weight parameters in the stDDM. Thus, once again, we checked that the method was able to correctly distinguish between different parameter magnitudes. We calculated the posterior probability distribution of the difference between $w_{h}$ parameters for different simulations. Figure S10 confirms that the method is able to distinguish between different magnitude of the weight parameters for the same attribute.

The piecewise constant averaging method also accurately estimates the difference in weights for the two separate attributes, $w_{h}-w_{t}$. As shown in [36], the probability of choosing one option over the other in a DDM framework can be approximated by logistic function. Thus, a change in unit of one attribute's weight relative to the other attribute's weight is quantifiable as the exponential of the difference in weights, e.g. $e^{w_{h}-w_{t}}$. Figure S11 and see Model (2) in 


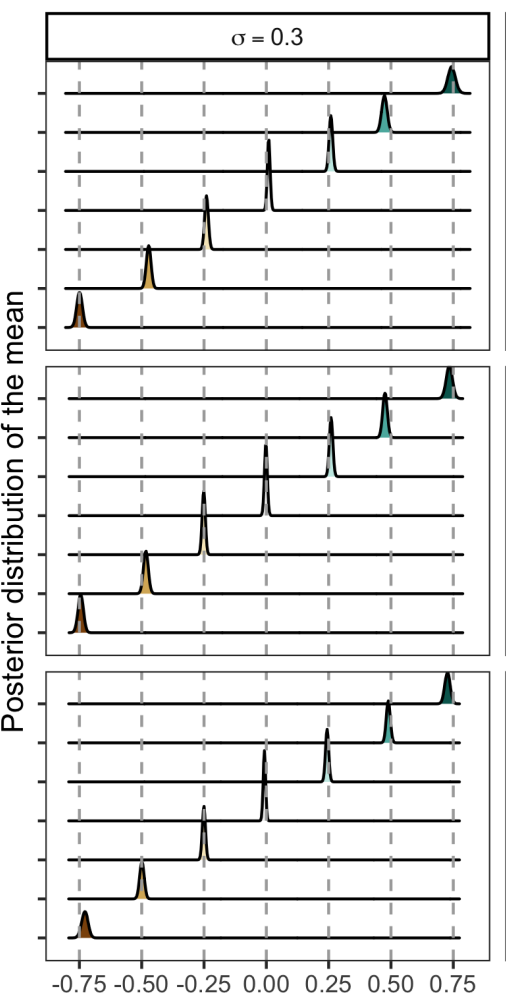

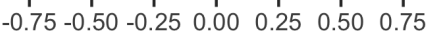
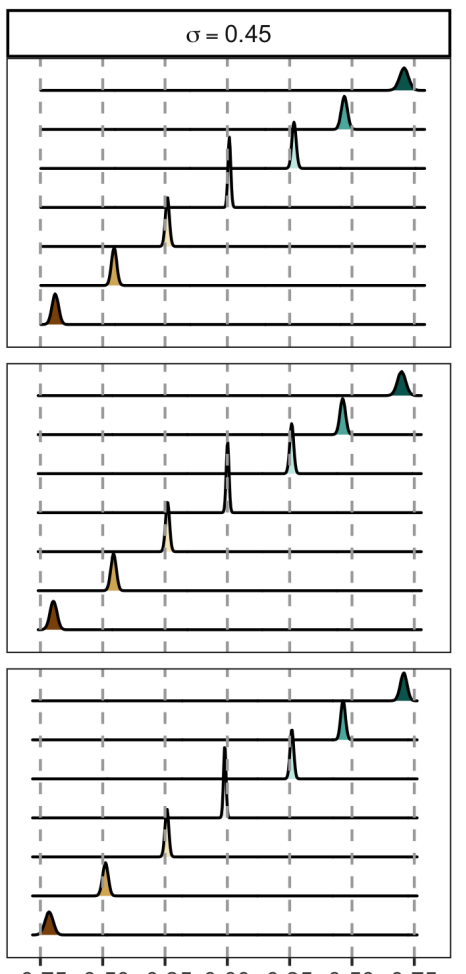

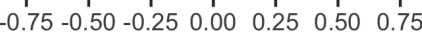

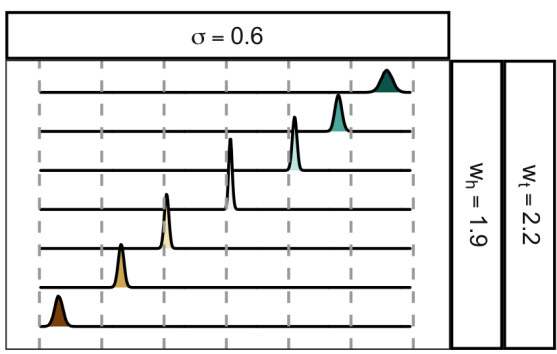

Generating s

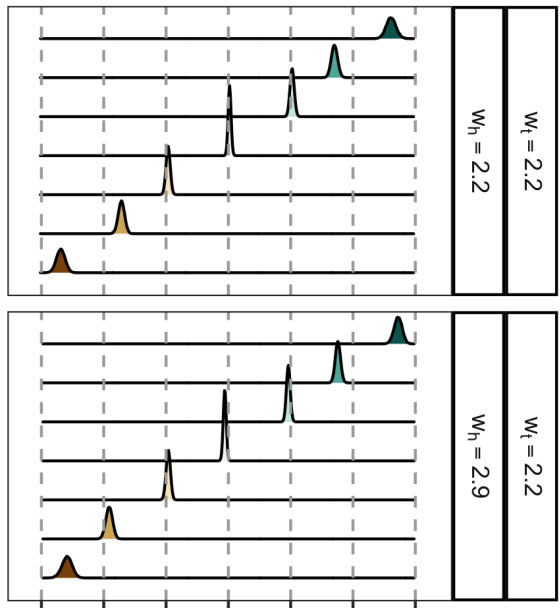

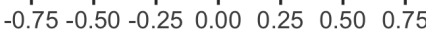

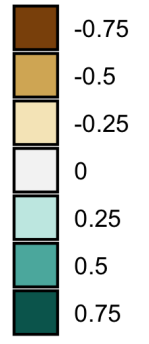

FIGURE 6 Hierarchical group level posterior probability distributions of the mean $s$ parameters from the HstDDM fits of simulations varying the weights and the $\sigma$ parameters. Each posterior distribution is based on a hierarchical Bayesian fit to 45 independent simulations. The dashed grey lines indicate the input generating parameters for the simulations.

Table S3 show that the piecewise constant averaging method is able to correctly recover the difference in weights $w_{h}-w_{t}$.

\section{| Fitting the HstDDM to groups of agents with heterogeneous model parameters}

Next, we tested parameter recovery accuracy for the HstDDM when each agent in a group has a different combination of model parameters. For each subject in the simulated groups, we randomly drew the parameters from gaussian distributions around a specific mean. The means and distributions of the generating parameters are shown in figure S5b. The model was able to accurately recover the generating parameters at the individual level when each subject had different generating parameters. Figure 8, S12 and S13 show the generating and estimated posterior distributions of the timing, weighting, and noise parameters at the individual level. The correlation between generating and estimated parameters further confirms the ability of the model to recover the parameters at the individual level in figure 7. 
(a)

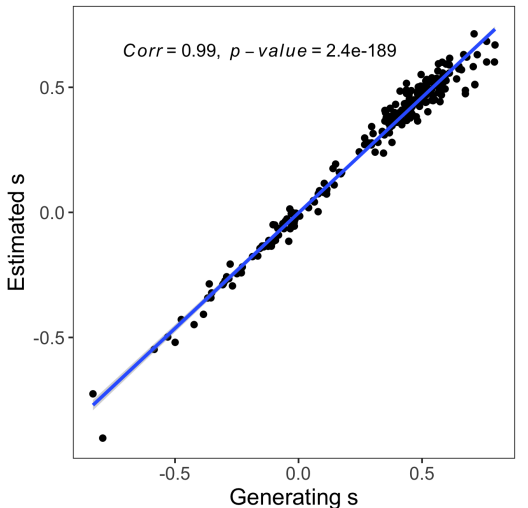

(c)

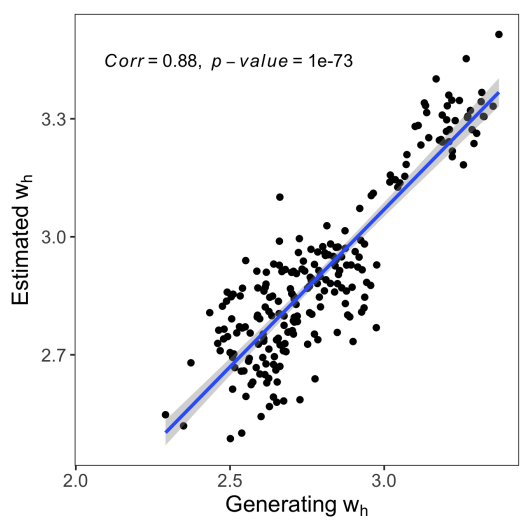

(b)

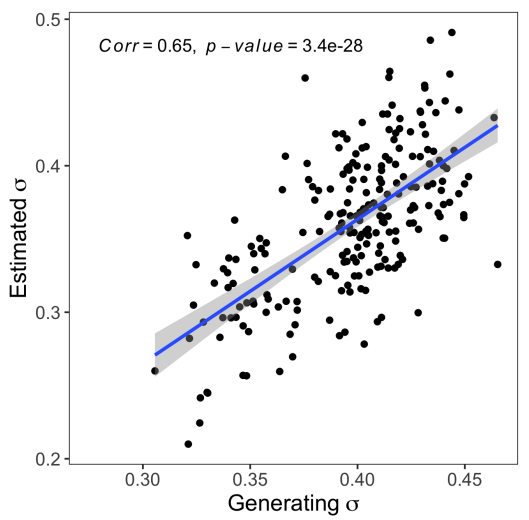

(d)

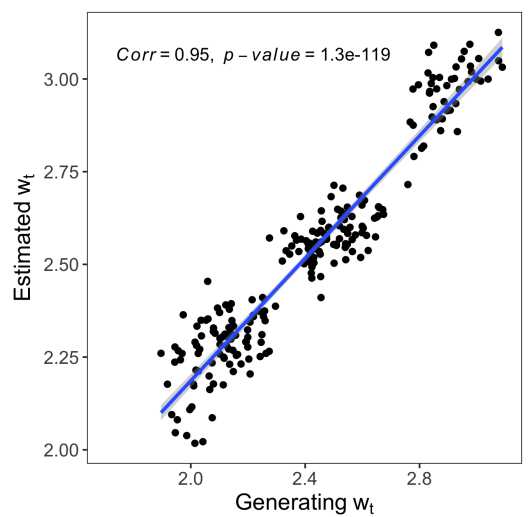

FIGURE 7 Correlation at the individual level between generating parameters and recovered parameters from the heterogeneous recovery fitting of the HstDDM. a) $s$ parameter. b) $\sigma$ parameter. c) $w_{h}$ parameter. d) $w_{t}$ parameter.

\subsection{Fitting empirical data from human participants}

Lastly, we report the results of fitting the HaDDM and HstDDM to two sets of human choice data using the piecewise constant averaging method. The full details of the experiments that generated these data are listed in the Methods section, and brief summaries of the behavioral paradigms are given in the two subsections below. Overall, simulations from the best-fitting HaDDM and HstDDM parameters can faithfully reproduce the human choice and response time patterns observed in the data they were fit to, indicating that the two models, when fit using the piecewise constant averaging method, fit human choice data well.

Similar to the recovery fitting analyses on simulated data above, we assumed that participants come from a single population, thus that the hierarchical structure consists of a single group. Specifically, we made the standard assumption that our human participants are members of a normally distributed population and assigned a uniform prior for each individual level parameter. 


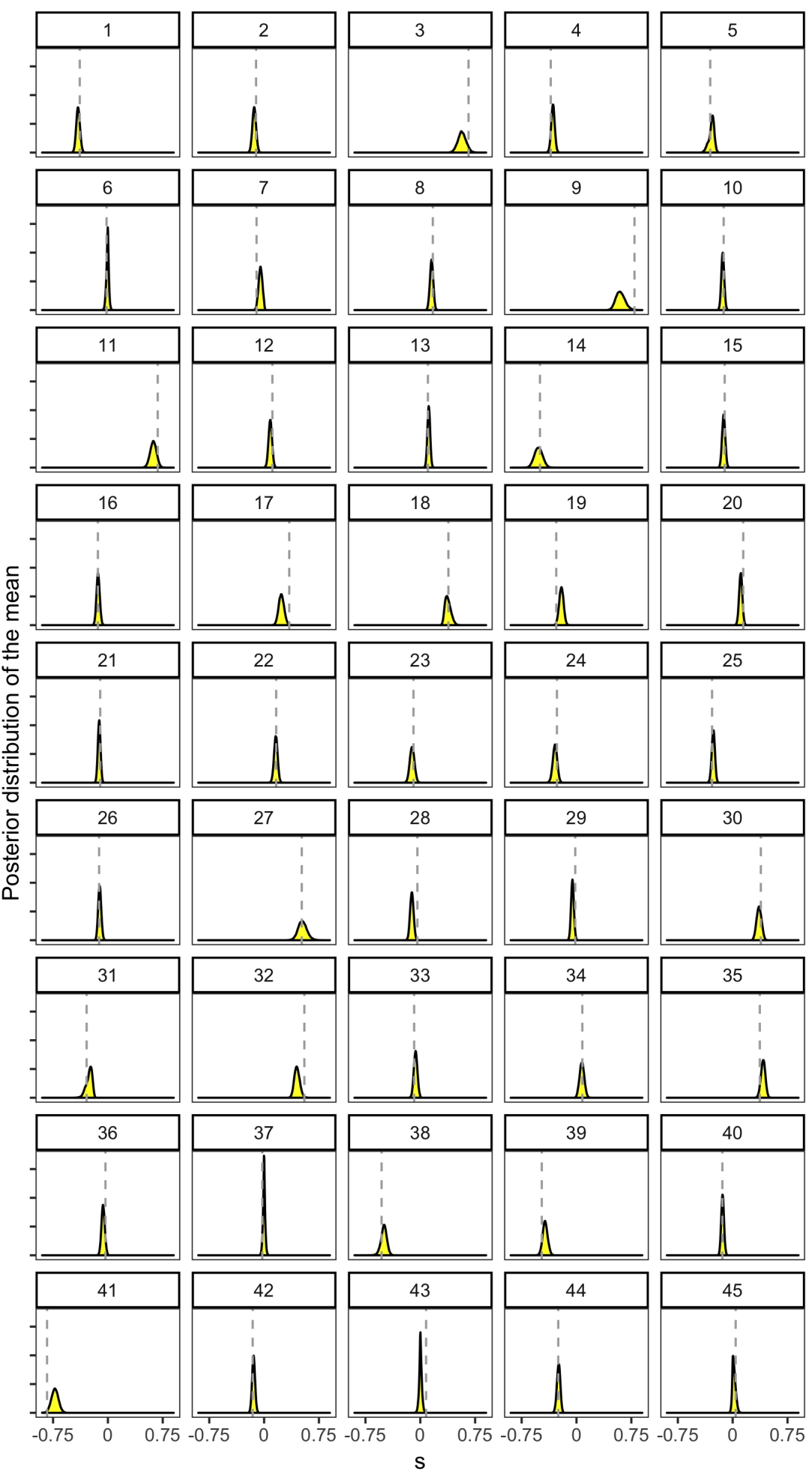

FIGURE 8 Individual level posterior probability distributions of the mean s parameters from the HstDDM fits for each subject in the data set. The dashed grey lines indicate the input generating parameters used for the simulations.

\section{| HaDDM fits to human choice data}

We used a set of empirical data from a binary lottery-choice task experiment in which participants had to complete 70 decision trials between a gamble and a sure option, to test the piecewise constant averaging method for fitting 
(a)

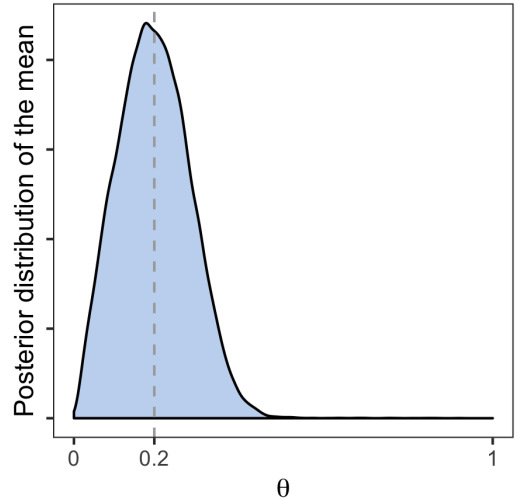

(c)

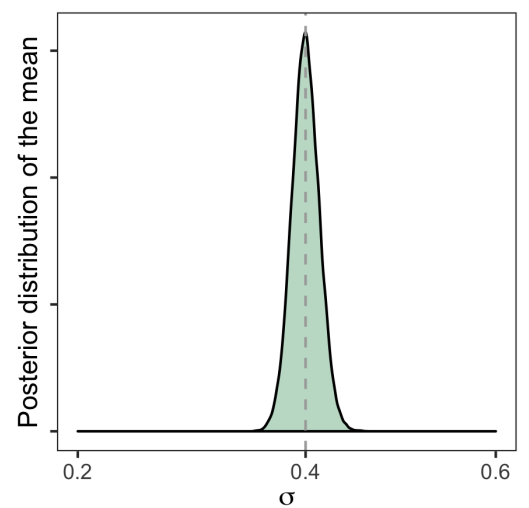

(b)

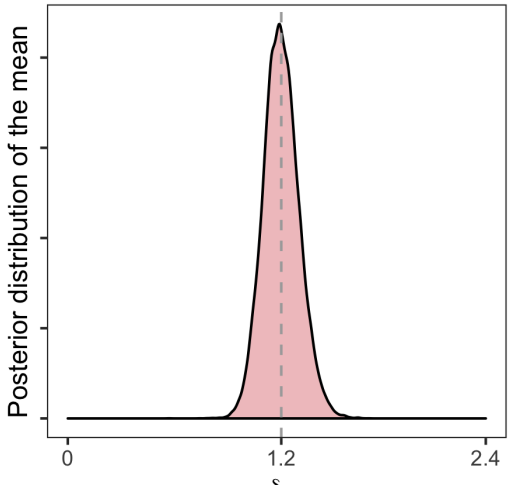

(d)

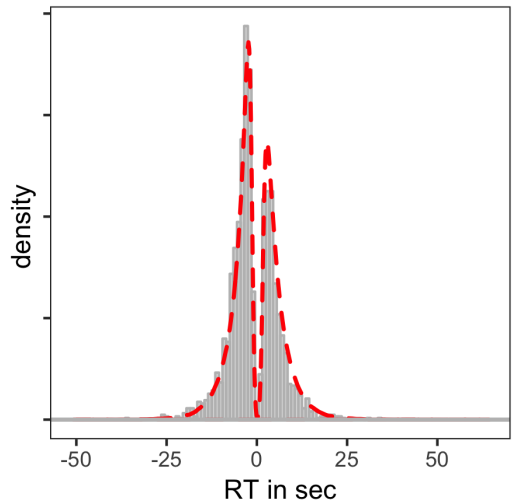

FIGURE 9 Model fitting analysis of experimental data with the HaDDM. a) Posterior probability distribution of the $\theta$ mean at the group level. b) Posterior probability distribution of the $\delta$ mean at the group level. c) Posterior probability distribution of the $\sigma$ mean at the group level. d) Goodness of fit plot of the reaction time for choosing the sure option (negative values) or the gamble (positive values). The red dashed line is the simulated reaction time with the mean of the posterior distribution of the fitted parameters for each individual, and the grey histogram indicates the empirical reaction time.

the aDDM. Briefly, in each trial of this task, the sure option offered a certain amount of money with probability $=1$, and the gamble option offered a higher amount with some probability $\mathrm{p}$ or nothing with probability 1-p. We tracked participants' eye-movements and recorded response times during each trial. Notably, participants were free to look anywhere on the computer screen that displayed the options and to take as long they needed to reach a decision (see Method section for further details).

The aDDM parameters estimated using the piecewise constant averaging method can accurately recreate the empirical pattern of choices and response times in the lottery data set. In particular, Figure $9 \mathrm{~d}$ shows that the simulated and the empirical reaction time distributions conditional to the choice are strikingly similar. Note that the simulations were performed using the mean of the posterior distribution for each individual for all fitted parameters. This suggests that the estimated $\delta, \theta$, and $\sigma$ parameters are reasonably accurate.

We ran an additional parameter recovery test after fitting the lottery task data set because the estimated values of certain parameters were outside the range we included in the a priori recovery tests above. Specifically, the posterior 
distribution of the $\delta$ parameter at the group level was outside of the range of values we used in the parameter recovery analysis in the previous section. The reason for the lower value of $\delta$ in these data is straightforward. The response times (see Figure 9d) in this particular lottery task experiment are slower than those in the food choice data upon which we based the parameter ranges in the parameter recovery analysis. Thus, the drift scaling parameters are estimated to be lower in the lottery choices to account for these slower RTs. Therefore, to make sure that lower drift scaling parameters do not affect the ability of the model to recover the discount factor parameter, $\theta$, we ran another recovery fitting analysis with the parameter values for $\delta$ and $\sigma$ that we obtained when fitting the model to the empirical data. We generated simulated choice data with the $\theta$ parameter set to $\{0.2,0.4,0.8\}$ in order to check whether the model was able to accurately recover the three values of $\theta$ when $\delta$ was 1.2. This additional parameter recovery test indicated that the piecewise constant averaging method was able to recover these known values of $\theta$ even with the smaller value of $\delta$ (see Figure 10a). Figure 11a-c further confirms the ability of the method and model to recover the fitted parameters for this specific range of parameters and subjects' option values.

(a)

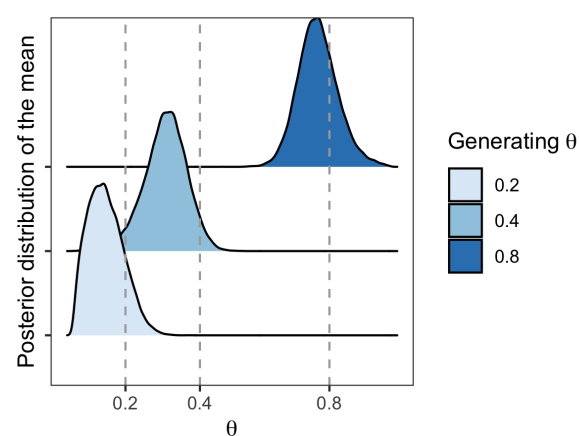

(b)

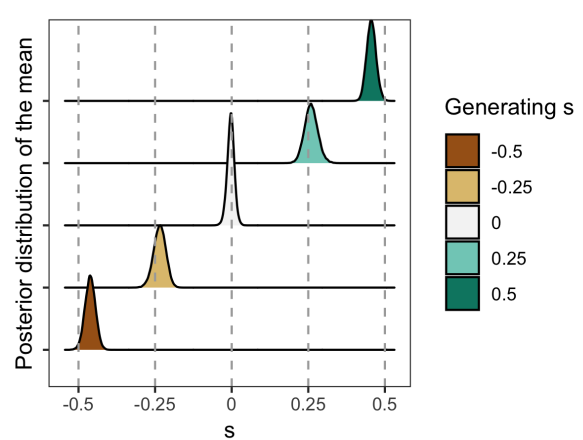

FIGURE 10 Group level posterior probability distributions of the mean. a) $\theta$ parameters from the HaDDM fits of simulations with $\delta$ and the $\sigma$ parameter values from the model fitting of the experimental data. The dashed grey lines indicate the input generating parameters for the simulations. b) $s$ parameters from the HstDDM fits of simulations with $w_{h}, w_{t}$ and the $\sigma$ parameter values from the model fitting of the experimental data. The dashed grey lines indicate the input generating parameters for the simulations. 
(a)

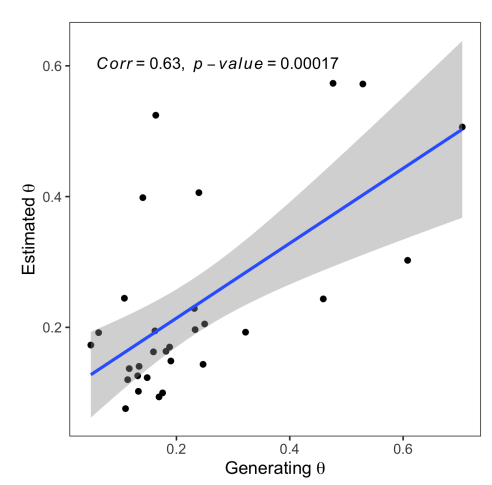

(d) (b)

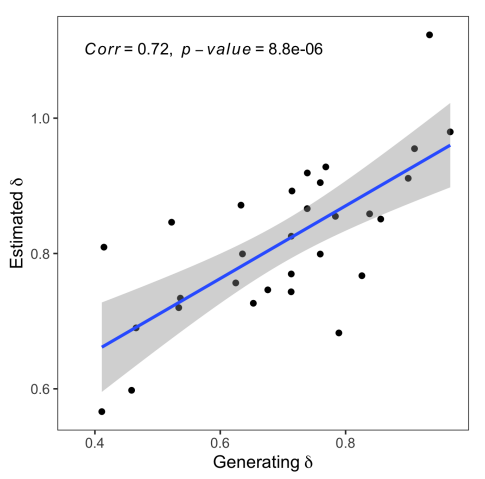

(c)

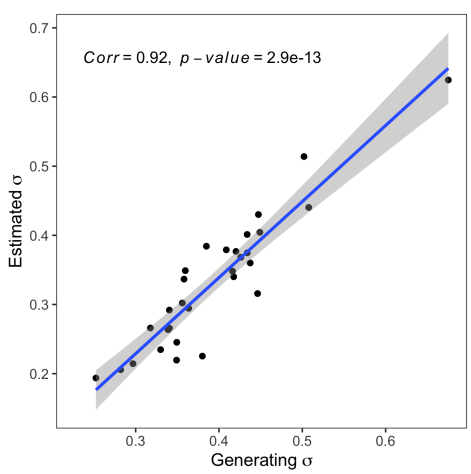

(e)
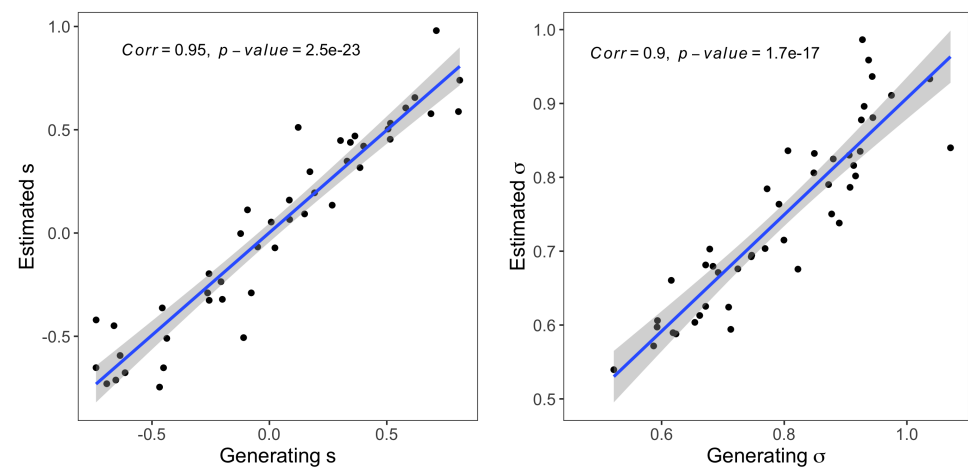

(f)

(g)
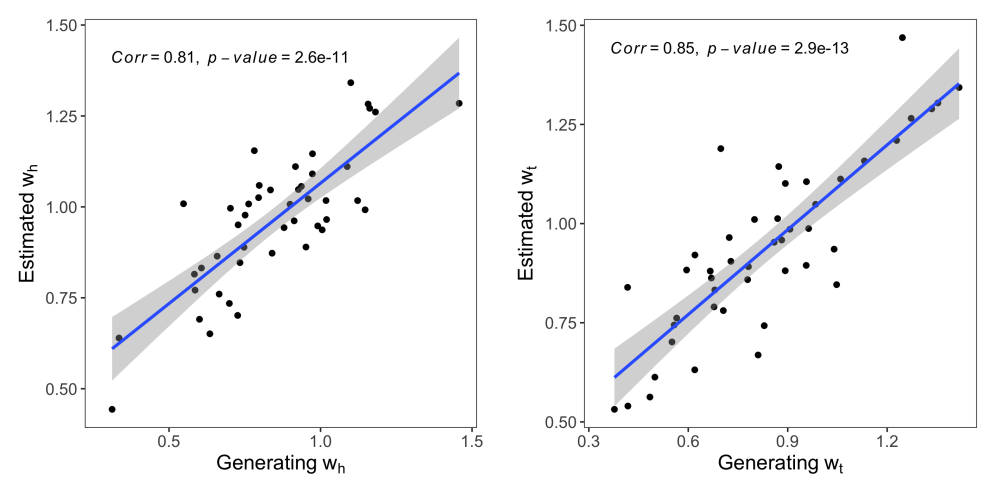

FIG URE 11 Correlation at the individual level between fitted parameters and recovered parameters from fitted empirical data with the HaDDM (from a to $\mathrm{c}$ ) and HstDDM (from $d$ to g). a) $\theta$ parameters from the HaDDM fits of simulations with parameter values from the model fitting of the experimental data. b) $\delta$ parameters from the $\mathrm{HaDDM}$ fits of simulations with parameter values from the model fitting of the experimental data. c) $\sigma$ parameters from the HaDDM fits of simulations with parameter values from the model fitting of the experimental data. d) $s$ parameters from the HstDDM fits of simulations with parameter values from the model fitting of the experimental data. e) $\sigma$ parameters from the HstDDM fits of simulations with parameter values from the model fitting of the experimental data. f) $w_{h}$ parameters from the HstDDM fits of simulations with parameter values from the model fitting of the experimental data. g) $w_{t}$ parameters from the HstDDM fits of simulations with parameter values from the model fitting of the experimental data. 


\section{| stDDM fits to human choice data}

To test the piecewise constant averaging method for the stDDM, we used real data from a binary food-choice task experiment in which participants had to complete 50 decision trials between food items. Before performing the food-choice task, participants had to rate all the 180 food items in the choice set for healthiness and tastiness (see Method section for details). Once again, we performed a confirmatory parameter recovery analysis similar to the one described for the aDDM above to make sure the model was able to return the true generating parameters for these specific data (see Figure 10b). Figure 11d-g shows the correlation between the simulated and the fitted parameters for this recovery analysis, further supporting the ability of the method to recover the parameters for this specific range of parameters and subjective option values. Figure 12 shows the results of fitting the HstDDM to this food-choice task data set for the drift-related parameters $\left(s, w_{h}, w_{t}\right.$, and $\left.\sigma\right)$. Panel $d$ in Figure 12 shows the simulated and the empirical reaction time distributions conditional to the choice. As for the HaDDM fits to the lottery choice data, HstDDM fits to food decisions produce choices and reaction times that are remarkably similar to the empirical ones.

(a)

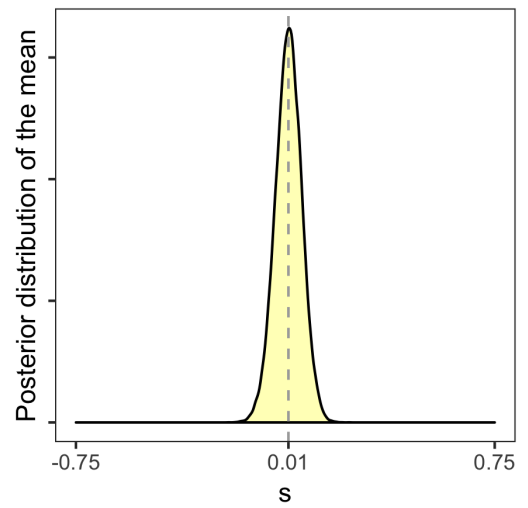

(c)

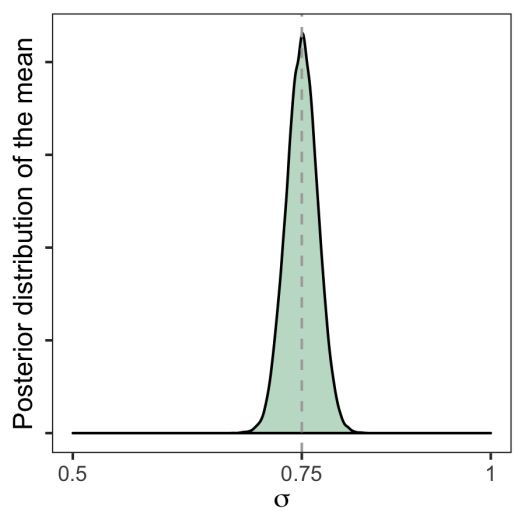

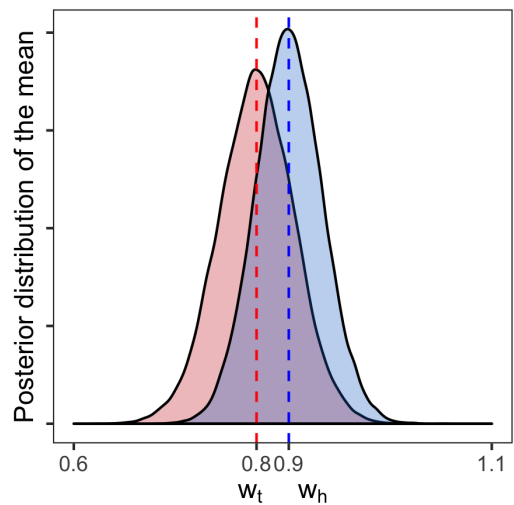

(d)

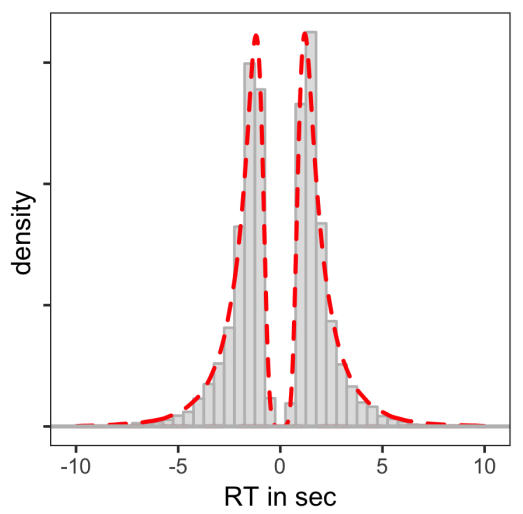

FIGURE 12 Model fitting analysis of experimental data with the HstDDM. a) Posterior probability distribution of the $s$ mean at the group level. b) Posterior probability distributions of the $w_{h}$ and $w_{t}$ means at the group level. c) Posterior probability distribution of the $\sigma$ mean at the group level. d) Goodness of fit plot of the reaction time for choosing the right option (negative values) or the left option (positive values). The red dashed line is the simulated reaction time with the fitted parameters and the grey histogram indicates the empirical reaction time. 


\section{| Computation time}

To give an estimate of the running time, we performed some additional fittings varying number of subjects and trials on a computer with the Ubuntu 16.04 operating system, 4 CPUs, and 16 GB of RAM. We ran 30 subjects with 70 trials each as a time baseline and measured the time increase when doubling number of trials, number of subjects or both. To estimate group-level and individual parameters in a data set of 30 subjects with 70 trials each, took 4522 seconds - about 75 minutes. When increasing the number of trials to 140 the time did not double, but only increased to 4789 seconds - about 80 minutes. When increasing the number of subjects to 60 - with 70 trials each - the time increased to 6472 seconds - about 108 minutes. And finally, when doubling both trials and subjects to 60 and 140, respectively, the time needed to fit the model was 8340 seconds - about 139 minutes.

\section{3 | DISCUSSION}

Here, we have presented the explicit derivation of a method to mathematically express a pcDDM as a standard DDM given the response time and the interval times in which the drift rate of the pcDDM is constant. We provided two practical examples of pcDDMs, the aDDM and the $\operatorname{stDDM}[15,16,1,18]$, and derived their mathematical formulations as standard DDMs. However, we note that this method is applicable to any time-varying drift rate for which one can compute the average drift rate over an observable period of time. Thus, this approach generalizes to a wide class of drift diffusion models.

We used several distinct parameter fitting and recovery analyses to show that this piecewise constant averaging method was able to accurately estimate the parameters of the aDDM and stDDMs. The first recovery analysis demonstrated the method for both the aDDM and the stDDM in their hierarchical Bayesian formulations was able to accurately estimate the different magnitudes of parameters at the group and individual levels in simulated data sets. The second recovery analysis showed that the method could accurately recover known heterogeneous individual-level parameters within the hierarchical Bayesian models. Finally, the last set of analyses showed the behavioral patterns in experimental data sets that we used as case studies could be accurately captured by the piecewise constant averaging method and that we could accurately recover the estimated empirical parameters.

Overall, all of the recovery fitting parameter analyses proved to be successful for both the HaDDM and the HstDDM with a high degree of accuracy. Specifically, we could apply this parameter estimation method to the two experimental data sets and show that the HaDDM and the HstDDM reproduced the choice and response time distributions well in both cases. Thus, the piecewise constant averaging method proved to be a simple, yet powerful, method that allows existing hierarchical Bayesian estimation tools developed for the standard DDM to also estimate the time-varying drift rate of pcDDMs. These findings are consistent with those of Cavanagh and colleagues [6], who tested different hypotheses about how visual fixations influence the DDM drift rate using a form of piecewise constant averaging in combination with a hierarchical Bayesian modeling framework [39]. We also showed how fast the model can be fit to experimental data of typical size. We believe this method will allow researchers not familiar with computational modelling to accurately fit typical experimental data sets. Nevertheless, we argue that any model fitting procedure applied to new data sets, even when methods or models have been tested to be robust and consistent in the field, should be used with caution, and few quality assurance steps should be followed rigorously before making any inference on the fitted parameters.

Running parameter recovery tests for the values estimated from a given empirical data set is good practice $[41,17$, 8]. It is especially important when dealing with data from novel experimental paradigms. It is often mathematically and computationally challenging to determine a priori all the potential combinations of parameter's values for which the 
model (or the fitting method) is not able to properly fit the data or return the true generating parameters. For instance, a low signal to noise ratio certainly affects the ability to accurately recover parameters, but what is "low" exactly? Further, a narrow range of value differences between choice options could affect the ability of a diffusion model to fit the data and derive the generating parameters, but what is a "good" or a "bad" range? Answering these questions with satisfactory precision is not trivial, and therefore, running a posteriori parameter recovery checks is best practice. The first step in such a recovery test is to check the ability of the model and fitting procedure to recover relevant known generating parameters. This can be done by simulating the data with the parameters obtained when originally fitting the empirical data, and then re-fitting the model to the simulated choice and response times. However, it is also possible that the recovery fitting fails by always returning the same parameters independently of the generating values. Therefore, success in this first step does not ensure you against this flaw in the model or fitting procedures. Thus, a necessary second step is to make sure that the model and fitting procedures are able to accurately distinguish between different generating parameter values. Specifically, for the second recovery testing step, all the simulating parameters but one are taken from the fitting of the empirical data as in the first step. The one remaining parameter is then varied across a range of plausible values that you want to test. This procedure ensures that the model can recover different magnitudes of that specific parameter of interest under the real data conditions, i.e. all the other parameters equal to the originally estimated values. Ideally, this second testing step would be run for each parameter in the model. At minimum, it should be run for all parameters of interest in a given study.

To conclude, we have shown that the piecewise constant averaging method is a simple, fast, general, and robust way to fit time-varying drift diffusion models within existing hierarchical Bayesian estimation frameworks. There has been a substantial increase in the postulation and application of time-varying sequential sampling models in recent years. Their ability to incorporate and quantify the effects of visual attention and other cognitive processes on decision outcomes makes them a useful advance over models that assume a constant rate of evidence accumulation. We hope that this tutorial on fitting such models in a simple, fast, and robust fashion will facilitate further application and refinement of time-varying DDMs in the decision sciences. 


\section{References}

[1] Dianna R Amasino et al. "Amount and time exert independent influences on intertemporal choice". In: Nature human behaviour 3.4 (2019), pp. 383-392.

[2] Sudeep Bhatia. "Associations and the accumulation of preference." In: Psychological review 120.3 (2013), p. 522.

[3] David H Brainard. "The psychophysics toolbox". In: Spatial vision 10.4 (1997), pp. 433-436.

[4] Scott D Brown and Andrew Heathcote. "The simplest complete model of choice response time: Linear ballistic accumulation". In: Cognitive psychology 57.3 (2008), pp. 153-178.

[5] Jerome R Busemeyer and James T Townsend. "Decision field theory: a dynamic-cognitive approach to decision making in an uncertain environment." In: Psychological review 100.3 (1993), p. 432.

[6] James F Cavanagh et al. "Eye tracking and pupillometry are indicators of dissociable latent decision processes." In: Journal of Experimental Psychology: General 143.4 (2014), p. 1476.

[7] Matthew J. Denwood. "runjags: An R Package Providing Interface Utilities, Model Templates, Parallel Computing Methods and Additional Distributions for MCMC Models in JAGS". In: Journal of Statistical Software 71.9 (2016), pp. 1-25. doi: 10.18637/jss.v071.i09.

[8] Nathan J Evans, Jennifer S Trueblood, and William R Holmes. "A parameter recovery assessment of time-variant models of decision-making”. In: Behavior research methods 52.1 (2020), pp. 193-206.

[9] William R Holmes. "A practical guide to the Probability Density Approximation (PDA) with improved implementation and error characterization". In: Journal of Mathematical Psychology 68 (2015), pp. 13-24.

[10] William R Holmes and Jennifer S Trueblood. "Bayesian analysis of the piecewise diffusion decision model". In: Behavior research methods 50.2 (2018), pp. 730-743.

[11] William R Holmes, Jennifer S Trueblood, and Andrew Heathcote. "A new framework for modeling decisions about changing information: The Piecewise Linear Ballistic Accumulator model”. In: Cognitive psychology 85 (2016), pp. 129.

[12] Jared M Hotaling, Jerome R Busemeyer, and Jiyun Li. "Theoretical developments in decision field theory: Comment on Tsetsos, Usher, and Chater (2010)." In: (2010).

[13] Ronald Hübner, Marco Steinhauser, and Carola Lehle. "A dual-stage two-phase model of selective attention." In: Psychological review 117.3 (2010), p. 759.

[14] M Kleiner, DH Brainard, and DG Pelli. "What is new in Psychophysics Toolbox". In: Perception 36 (2007).

[15] Ian Krajbich, Carrie Armel, and Antonio Rangel. "Visual fixations and the computation and comparison of value in simple choice". In: Nature neuroscience 13.10 (2010), p. 1292.

[16] Ian Krajbich and Antonio Rangel. "Multialternative drift-diffusion model predicts the relationship between visual fixations and choice in value-based decisions". In: Proceedings of the National Academy of Sciences 108.33 (2011), pp. 1385213857.

[17] Michael D Lee et al. "Robust modeling in cognitive science". In: Computational Brain \& Behavior 2.3-4 (2019), pp. 141153.

[18] Silvia U Maier et al. "Dissociable mechanisms govern when and how strongly reward attributes affect decisions". In: Nature Human Behaviour (2020), pp. 1-15.

[19] In Jae Myung. "Tutorial on maximum likelihood estimation". In: Journal of mathematical Psychology 47.1 (2003), pp. 90100.

[20] Daniel J Navarro and lan G Fuss. "Fast and accurate calculations for first-passage times in Wiener diffusion models". In: Journal of Mathematical Psychology 53.4 (2009), pp. 222-230.

[21] Denis G Pelli. "The VideoToolbox software for visual psychophysics: Transforming numbers into movies". In: Spatial vision 10.4 (1997), pp. 437-442. 
[22] Martyn Plummer et al. "JAGS: A program for analysis of Bayesian graphical models using Gibbs sampling". In: Proceedings of the 3rd international workshop on distributed statistical computing. Vol. 124. 125.10. Vienna, Austria. 2003, pp. 110.

[23] Martyn Plummer. rjags: Bayesian Graphical Models using MCMC. R package version 4-10. 2019. url: https : //CRAN . Rproject .org/package=rjags.

[24] R Core Team. R: A Language and Environment for Statistical Computing. R Foundation for Statistical Computing. Vienna, Austria, 2020. url: https://www.R-project.org/.

[25] Roger Ratcliff. "A theory of memory retrieval." In: Psychological review 85.2 (1978), p. 59.

[26] Roger Ratcliff and Philip L Smith. "A comparison of sequential sampling models for two-choice reaction time." In: Psychological review 111.2 (2004), p. 333.

[27] Roger Ratcliff, Anjali Thapar, and Gail McKoon. "The effects of aging on reaction time in a signal detection task." In: Psychology and aging 16.2 (2001), p. 323.

[28] Robert M Roe, Jermone R Busemeyer, and James $T$ Townsend. "Multialternative decision field theory: A dynamic connectionst model of decision making." In: Psychological review 108.2 (2001), p. 370.

[29] Vaibhav Srivastava et al. "A martingale analysis of first passage times of time-dependent Wiener diffusion models". In: Journal of mathematical psychology 77 (2017), pp. 94-110.

[30] Brandon M Turner and Per B Sederberg. "A generalized, likelihood-free method for posterior estimation". In: Psychonomic bulletin \& review 21.2 (2014), pp. 227-250.

[31] Brandon M Turner and Per B Sederberg. "Approximate Bayesian computation with differential evolution". In: Journal of Mathematical Psychology 56.5 (2012), pp. 375-385.

[32] Marius Usher and James L McClelland. "Loss aversion and inhibition in dynamical models of multialternative choice." In: Psychological review 111.3 (2004), p. 757.

[33] Joachim Vandekerckhove, Francis Tuerlinckx, and Michael D Lee. "Hierarchical diffusion models for two-choice response times." In: Psychological methods 16.1 (2011), p. 44.

[34] Dominik Wabersich and Joachim Vandekerckhove. "Extending JAGS: A tutorial on adding custom distributions to JAGS (with a diffusion model example)". In: Behavior research methods 46.1 (2014), pp. 15-28.

[35] Dominik Wabersich and Joachim Vandekerckhove. "The RWiener package: An R package providing distribution functions for the Wiener diffusion model”. In: The R Journal 6.1 (2014). R package version 1.3-2, pp. 49-56.

[36] Ryan Webb. "The (neural) dynamics of stochastic choice". In: Management Science 65.1 (2019), pp. 230-255.

[37] Corey N White et al. "Using diffusion models to understand clinical disorders". In: Journal of Mathematical Psychology 54.1 (2010), pp. 39-52.

[38] Hadley Wickham. ggplot2: Elegant Graphics for Data Analysis. Springer-Verlag New York, 2016. isbn: 978-3-319-242774. url: https://ggplot2.tidyverse.org.

[39] Thomas V Wiecki, Imri Sofer, and Michael J Frank. "HDDM: hierarchical bayesian estimation of the drift-diffusion model in python". In: Frontiers in neuroinformatics 7 (2013), p. 14.

[40] Claus O. Wilke. ggridges: Ridgeline Plots in 'ggplot2'. R package version 0.5.2. 2020. url: https : //CRAN . R-project. org/ package =ggridges.

[41] Robert C Wilson and Anne GE Collins. "Ten simple rules for the computational modeling of behavioral data". In: Elife 8 (2019), e49547. 


\section{4 | MATERIALS AND METHODS}

\section{1 | Datasets}

\section{| Lottery task}

Subjects. Thirty-six healthy subjects participated in this experiment. Six of them were excluded from the experiment because they failed to understand the choice task based on a priori performance criteria for the comprehension questions. Subjects received monetary compensation for their participation, were informed about all aspects of the experiment and gave written informed consent. The experiments conformed to the standards of the Declaration of Helsinki and the Human Subjects Committee of the University of Zürich approved the experimental protocol.

Task. The experimental design was divided into two main parts: a decision phase and an elicitation phase. During this decision phase, participants completed 70 trials in which they made lottery decisions between a sure option and a gamble. At the beginning of each trial the participant was presented with a monetary endowment (e.g. 'You receive $50 \mathrm{CHF}^{\prime}$ ). Ten different starting amounts were used in the experiment (from $10 \mathrm{CHF}$ to $100 \mathrm{CHF}$ with an increment of $10 \mathrm{CHF}$ ). To move on to the decision screen, the participants had to fixate for 2 seconds on the received monetary amount, which appeared in the centre of the screen after the choice was indicated. After the outcome fixation period was completed, a new trial began with a sure option and a gamble appearing on the screen. The sure option was presented as an amount of money retained from the starting amount (e.g. keep $20 \mathrm{CHF}$ out of a total of $50 \mathrm{CHF}$ ). The gamble option always offered the chance to keep all of the starting amount of money with some probability. Seven different probabilities were used in the study, such that the probability of winning in a given trial was either $30 \%, 40 \%$, $50 \%, 60 \%, 70 \%, 80 \%$ or $90 \%$. The expected outcomes, i.e. expected value (EV), of sure and gamble options were always equivalent in each trial. However, the EV varied across trials from 3 to 90 CHF (approximately 3 - 90 USD). The sure and the gamble options on the screen were represented through partially or completely filled rectangles and pie-charts. The proportion of the rectangles filled with color indicated the amount of money that the subject could keep from the starting amount, while the proportion of the pie charts filled with color represented probabilities (e.g. the sure option always had a pie-chart completely filled with a color). Before performing the choice task, subjects went through a training task to learn the meaning of rectangles and pie-charts.

In the second phase of the experiment, the participants performed a certainty equivalence (CE) elicitation task in which they were shown all the $\mathbf{7 0}$ gambles that they encountered during the choice task. In each trial, for a total of 70 trials, a gamble and a list of sure amounts of money was shown to the participant. The subject had to make 10 decisions in each trial between the gamble displayed on the left hand-side of the screen and 10 sure amounts of money. The sure amounts of money were displayed in decreasing order from a maximum value smaller but close to the amount of money that they could win if they choose the gamble, to a minimum value close to zero. For instance, a gamble can be described as follows. You first receive an endowment of $100 \mathrm{CHF}$ and you have a probability of $70 \%$ of keeping all the $100 \mathrm{CHF}$ (30\% of receiving nothing). In the first line, the participant had to decide between the gamble and a sure amount of $95 \mathrm{CHF}$. In the second line, she had to decide between the gamble and a sure amount of $85 \mathrm{CHF}$, and so on until the last line in which she had to choose between the gamble and a sure amount of money of $5 \mathrm{CHF}$. The CE was calculated as the mean between the two amounts of money where the subject switches from choosing the sure amount to choosing the gamble. Thus, in the example above, if a participant chooses the sure amounts of money for every decision lines until $55 \mathrm{CHF}$, and she chooses the gamble from $45 \mathrm{CHF}$ on, the CE for this gamble is 50 CHF. Participants were only allowed to switch from choosing the sure amount to choosing the gamble a maximum of once per trial - including the possibility of never switching, i.e. always choosing the gamble or always choosing 
the sure amount.

All tasks were programmed in Matlab 2015b (Matworks), using the Psychophysics Toolbox extension [3, 21, 14].

Eye Tracking. Before each decision trial, subjects were required to fixate the monetary endowment positioned at the center of the screen for $2 \mathrm{~s}$ before the options would appear, ensuring that subjects began every trial fixating on the same location. Subject's gazes were recorded at $500 \mathrm{~Hz}$ with an EyeLink-1000 (http://www.sr-research.com/) eye tracker. Choice trials with no gaze time on any option attribute were excluded from the analysis (17 trials, $0.004 \%$ of the pooled data from the 30 subjects).

\section{| Food-choice task}

Subjects. Ninety-seven healthy subjects participated in this experiment. Eleven of them were excluded from the experiment because they either failed the questionnaire about the choice task - i.e. understanding the task, or because they did not comply with the health agreement (described below). Subjects received monetary compensation and a food reward for their participation, were informed about all aspects of the experiment and gave written informed consent.

Task. The experimental design was divided into two parts: a rating phase and a decision phase. During the rating phase participants rated 180 food items for healthiness and tastiness on a continuous scale with the anchors - 5 (bad) to 5 (good). The food items were shown to the subjects as images on the computer screen with a rating bar below one at the time. After the ratings, subjects performed a binary food-choice task consisting of 60 trials in which two food items were presented on the computer screen and they had to choose the food that they would like to receive at the end of the experiment. Before starting the food-choice task, participants read through a health agreement form in which the benefits of eating healthy were explained and in which subjects were asked to try to make healthy choices during the following food-choice task. After reading the form, subjects had to express their willingness to try to eat, i.e. choose, healthier foods by check-marking the yes or no option on the health agreement form. Participation in the study and receipt of the monetary and food compensation were not conditional on agreeing to try and make healthy choices. However, subjects who were not willing to try to eat healthy were subsequently excluded from the data analysis.

\section{| Simulated choice data}

For all simulated agents we assigned subjective values for the gambles or food items based on the empirical data from the human subjects described above. In the case of the aDDM, we used empirical fixation patterns as well. If a simulated aDDM trial did not cross the upper or lower boundary before the end of the empirical fixation pattern with which it was paired, then we drew additional fixations from the empirical distribution of fixation durations for the gamble or sure option until the simulated evidence accumulation process crossed a boundary. For each parameter combination in the aDDM, we simulated 30 agents that each faced 70 trials of unique subjective values and fixation patterns. For each parameter combination in the stDDM we simulated 45 agents that each faced 60 trials with unique subjective values. 


\section{2 | Model fitting}

We fitted and simulated data with R [24] using the runjags [22, 23, 7], RWiener [35] packages, and we analyzed and plot the data with ggplot2 [38] and ggridges [40]. We wrote the drift diffusion models in JAGS using the jags-wiener module [34]. This function takes as inputs the following parameters: ( $\alpha$, ndt, $\beta, \mu, \sigma)$. The parameter $\alpha$ is the boundary separation parameter, ndt the non-decision time, $\beta$ the bias parameter, $\mathrm{d}$ the drift rate parameter and $\sigma$ the standard deviation of the drift process. Concerning the drift rate $\mu$, we set it to be equal to the function derived in equations 7 and 9 above for the aDDM and the stDDM, respectively. We repeat those equations here in this section for the reader's convenience. Thus, for the aDDM the drift rate function was set to

$$
\mu=\frac{\tau_{S}}{\tau} \delta\left(V_{S}-\theta V_{G}\right)+\frac{\tau_{G}}{\tau} \delta\left(\theta V_{S}-V_{G}\right)
$$

where $\tau$ is the reaction time, $\tau_{S}$ and $\tau_{G}$ the total fixation time towards the sure option and the gamble respectively, $\delta$ is the drift constant parameter, and $v_{S}$ and $v_{G}$ the value of the sure option and the gamble, respectively.

For the stDDM, the drift rate function was set to

$$
\mu= \begin{cases}\frac{s}{\tau} w_{T} \mathrm{VD}_{T}+\frac{\tau-s}{\tau}\left(w_{H} \mathrm{VD}_{H}+w_{T} \mathrm{VD}_{T}\right) & \text { if } s>0 \wedge s<\tau \\ \frac{|s|}{\tau} w_{H} \mathrm{VD}_{H}+\frac{\tau-|s|}{\tau}\left(w_{H} \mathrm{VD}_{H}+w_{T} \mathrm{VD}_{T}\right) & \text { if } s<0 \wedge|s|<\tau \\ w_{T} \mathrm{VD}_{T} & \text { if } s>0 \wedge s>\tau \\ w_{H} \mathrm{VD}_{H} & \text { if } s<0 \wedge|s|>\tau\end{cases}
$$

where $w_{T}$ is the weight given to the taste attribute, $w_{H}$ the weight to the health attribute, $\mathrm{VD}_{T}$ and $\mathrm{VD}_{H}$ are the value differences in taste and in health respectively, $s$ is the time at which the health attribute comes into the accumulation process $-s>0$ means taste is on from the beginning and health comes in at time $s, s<0$ means that the health attribute is on the accumulation process from the beginning and taste comes in at time $|s|$.

For both models, we fixed the boundary separation parameter $\beta=2$, and estimated the standard deviation of the noise $\sigma$ and the non-decision time ndt. Specifically for the aDDM, we estimated the drift rate constant $\delta$ and the discount factor $\theta$, whereas the total fixation times $\tau_{S}$ and $\tau_{G}$ were given as an input to the model. Concerning the stDDM, we estimated the weights parameters $w_{T}$ and $w_{H}$, and the relative starting time parameter $s$.

For the hierarchical bayesian fitting, we drew 10,000 posterior samples after an initial burn-in of 50,000 samples for each chain for a total of three chains. The convergence of the model was assessed every time it was run in the following ways. We examined the traceplot for each single parameter, a visual monitoring the convergence of the three chains. In addition, we monitored the convergence diagnostic factor (psrf), also known as the Gelman and Rubin's potential scale reduction factor, $\hat{R}$, which provides an estimate of convergence based on the variance of an estimated parameter between chains, and the variance within a chain." In the hierarchical models, we used uniform priors for each parameter that were broad with respect to the plausible range of that parameter. We assumed that the participants (or simulated agents) came from a single population, and thus the hierarchical structure consisted of a single group. In other words, we made the standard assumption that the separate individuals are members of a normally distributed population.

The $R$ and JAGS code containing all details of the data fitting and simulations for this paper as well as the empirical data from the lottery and food choice experiments can be found at https://github. com/galombardi/method_ HtSSM_aDDM. 


\section{5 | SUPPLEMENTARY MATERIAL}

\section{1 | Figures}
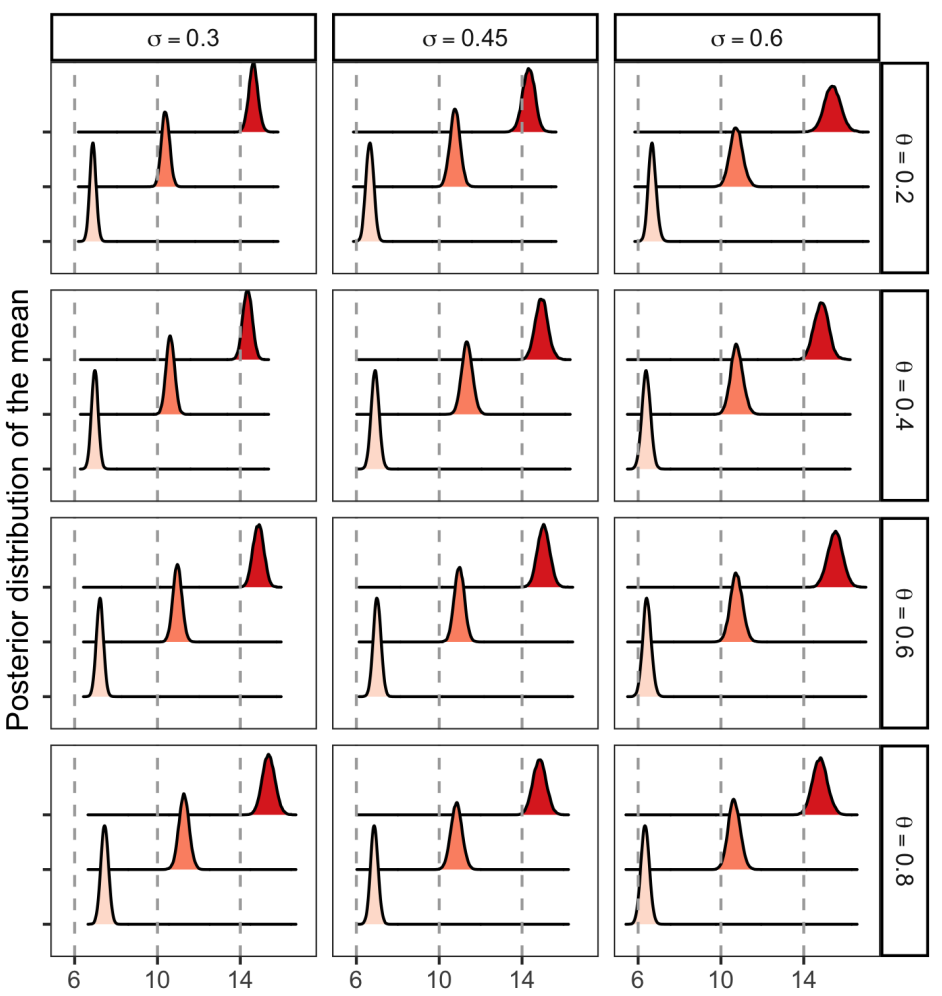

Generating $\delta$
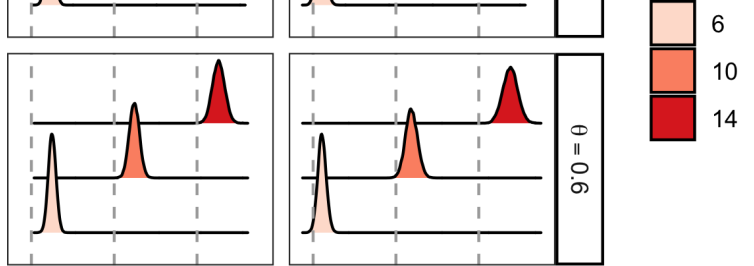

FIGURE S1 Group level posterior probability distributions of the mean $\delta$ parameters from the HaDDM fits of simulations varying the $\theta$ and the $\sigma$ parameters. Each posterior distribution is based on a hierarchical Bayesian fit to 30 independent simulations. The dashed grey lines indicate the input generating parameters for the simulations. 

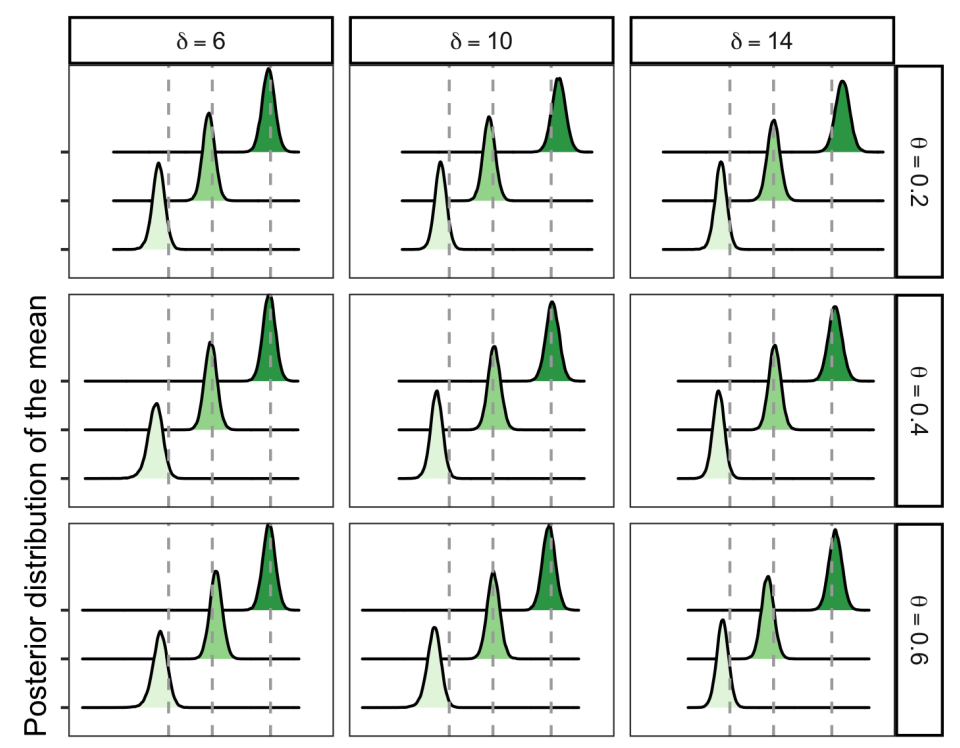

Generating $\sigma$
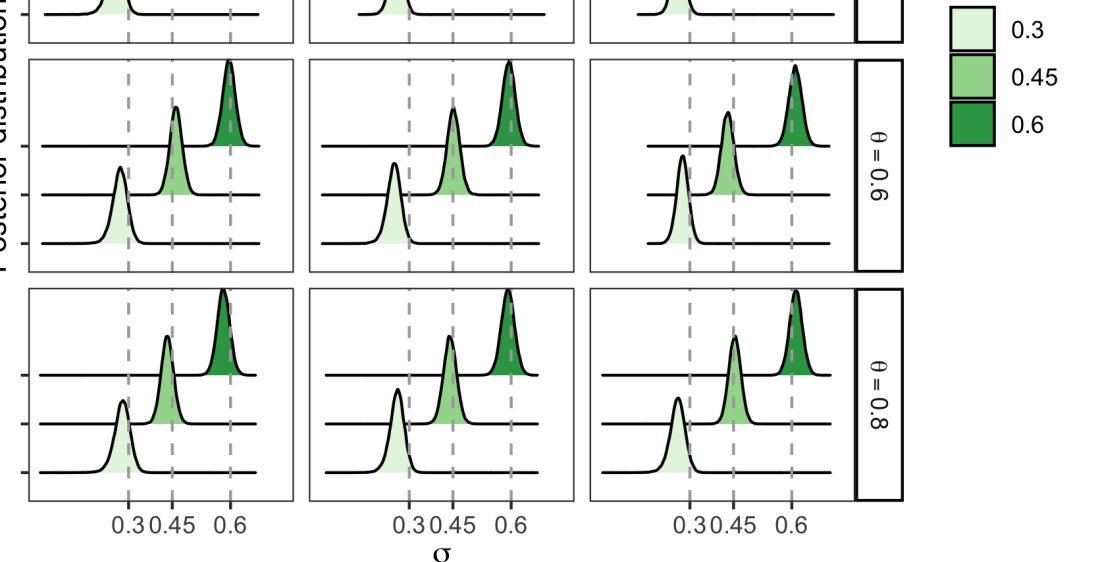

FIGURE S2 Group level posterior probability distributions of the mean $\sigma$ parameters from the HaDDM fits of simulations varying the $\delta$ and the $\theta$ parameters. Each posterior distribution is based on a hierarchical Bayesian fit to 30 independent simulations. The dashed grey lines indicate the input generating parameters for the simulations. 
(a)
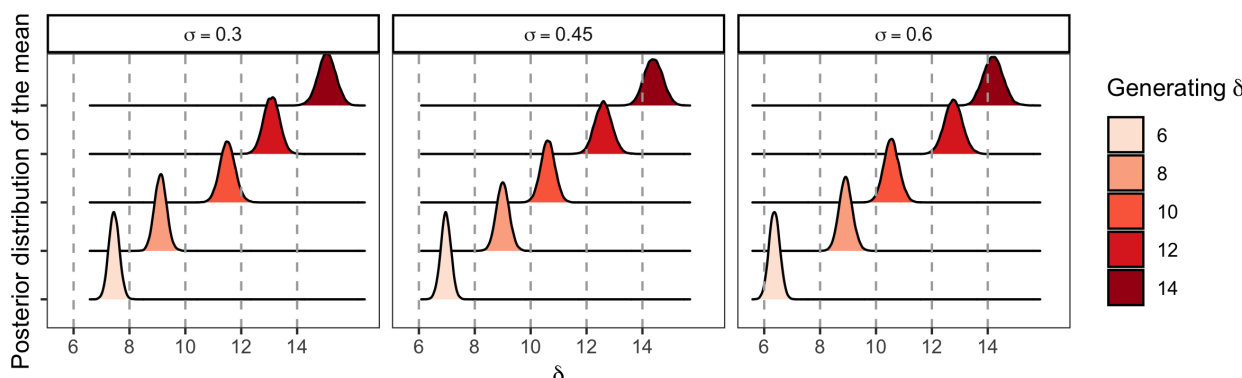

(b)
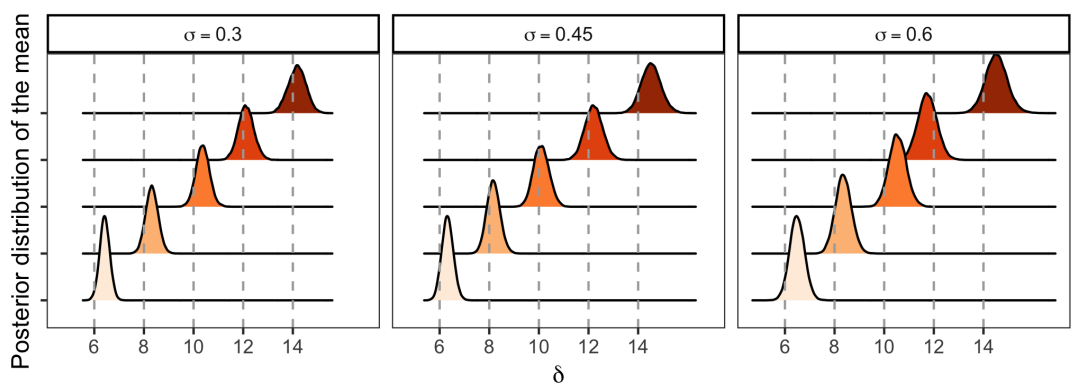

Generating $\delta$

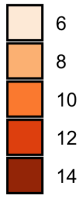

FIGURE S3 Group level posterior probability distributions of the $\delta$ mean from HDDM fits of separate simulations. Each posterior distribution is based on a hierarchical Bayesian fit to 30 independent simulations. The dashed grey lines indicate the input generating parameter. a) Simulations were performed with the discrete bounded accumulation series of the DDM - i.e. Euler method. b) Simulations were performed with the continuous-time SDE version of the DDM 


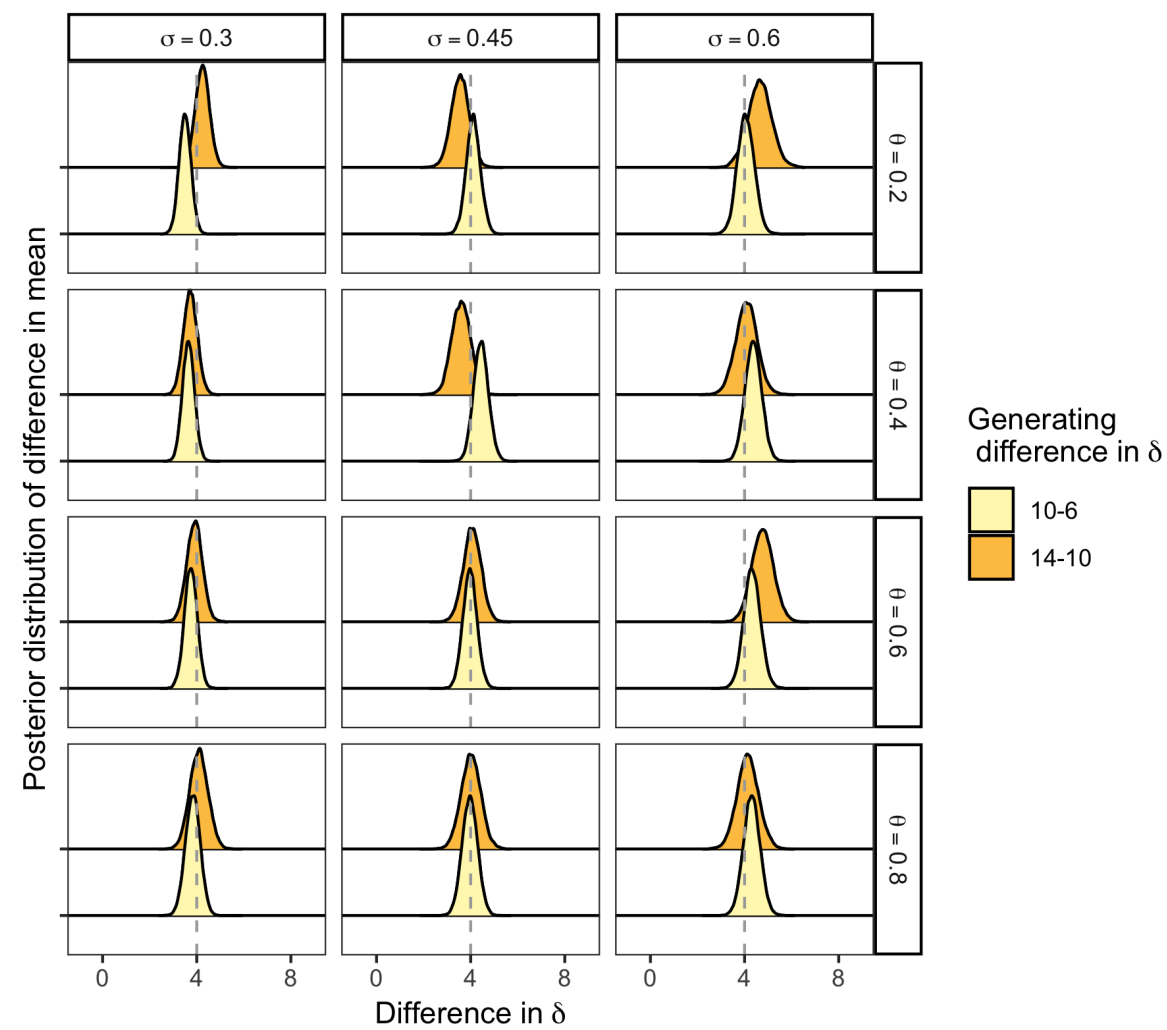

FIGURE S4 Group level posterior probability distributions of the difference in mean between $\delta$ parameters from HaDDM fits of separate simulations varying the $\theta$ and the $\sigma$ parameters. Each posterior difference distribution is based on a hierarchical Bayesian fits to two sets of 30 independent simulations. The dashed grey lines indicate the input generating parameter difference between simulations. 
(a)

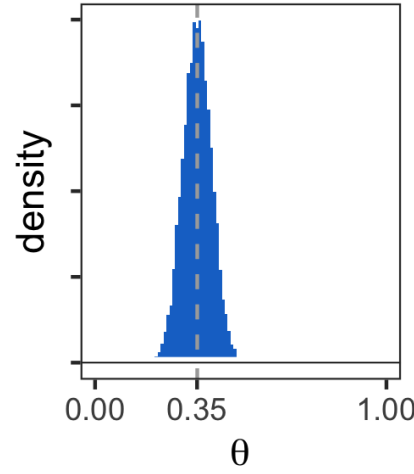

(b)

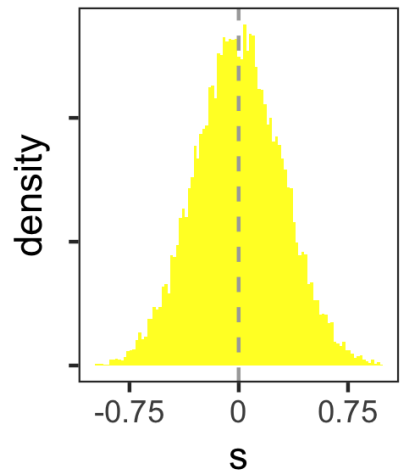

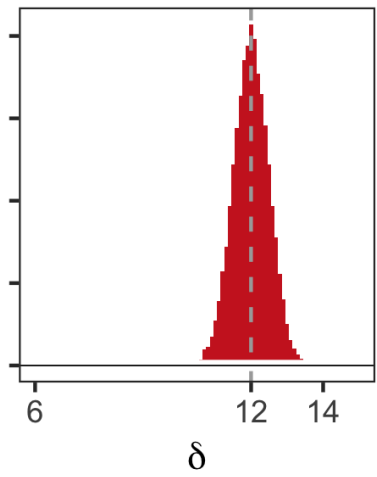
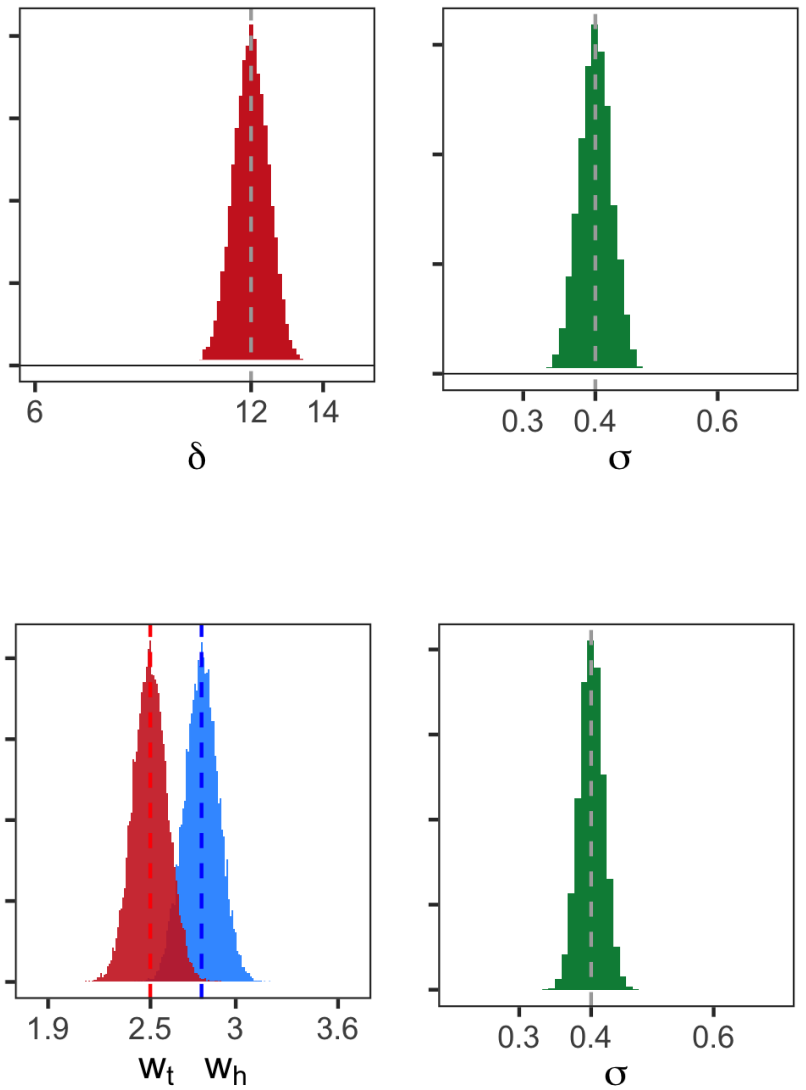

FIGURE S5 Distributions for the generating parameters in used the parameter recovery analyses for heterogeneous groups. a) Generating gaussian distributions $\mathrm{N}\left(\mu, \sigma^{2}\right)$ of the aDDM parameters. $\theta \sim \mathrm{N}(0.35,0.0025)$, $\delta \sim \mathrm{N}(12,0.25), \sigma \sim \mathrm{N}(0.41,0.00064)$. b) Generating gaussian distributions $\mathrm{N}\left(\mu, \sigma^{2}\right)$ of the stDDM parameters. $s \sim \mathrm{N}(0,0.09), w_{t} \sim \mathrm{N}(2.5,0.01), w_{h} \sim \mathrm{N}(2.8,0.01), \sigma \sim \mathrm{N}(0.41,0.0006)$. 


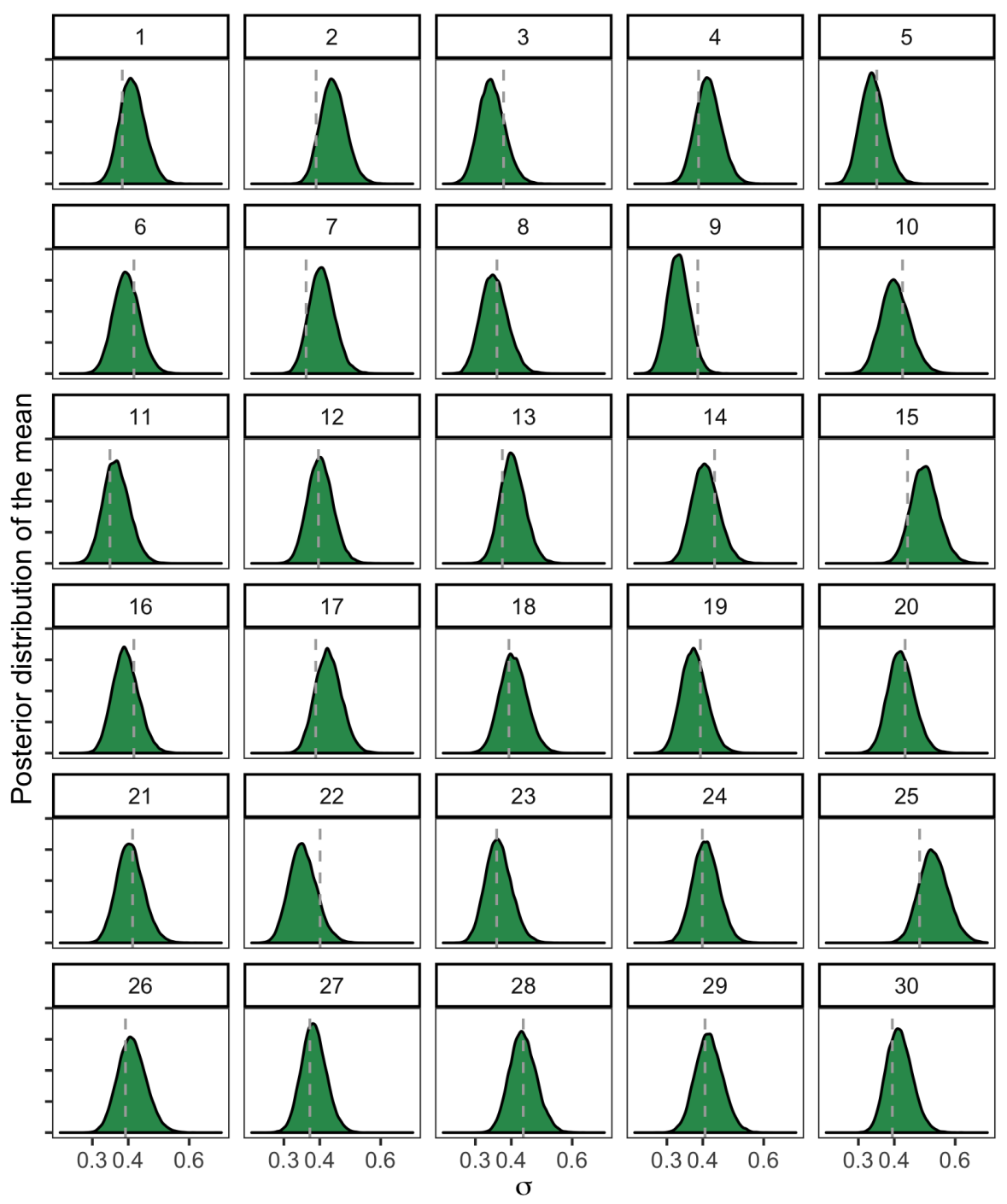

FIGURE S6 Individual level posterior probability distributions of the mean $\sigma$ parameters from the HaDDM fits for each subject in the data set. The dashed grey lines indicate the input generating parameters used for the simulations. 

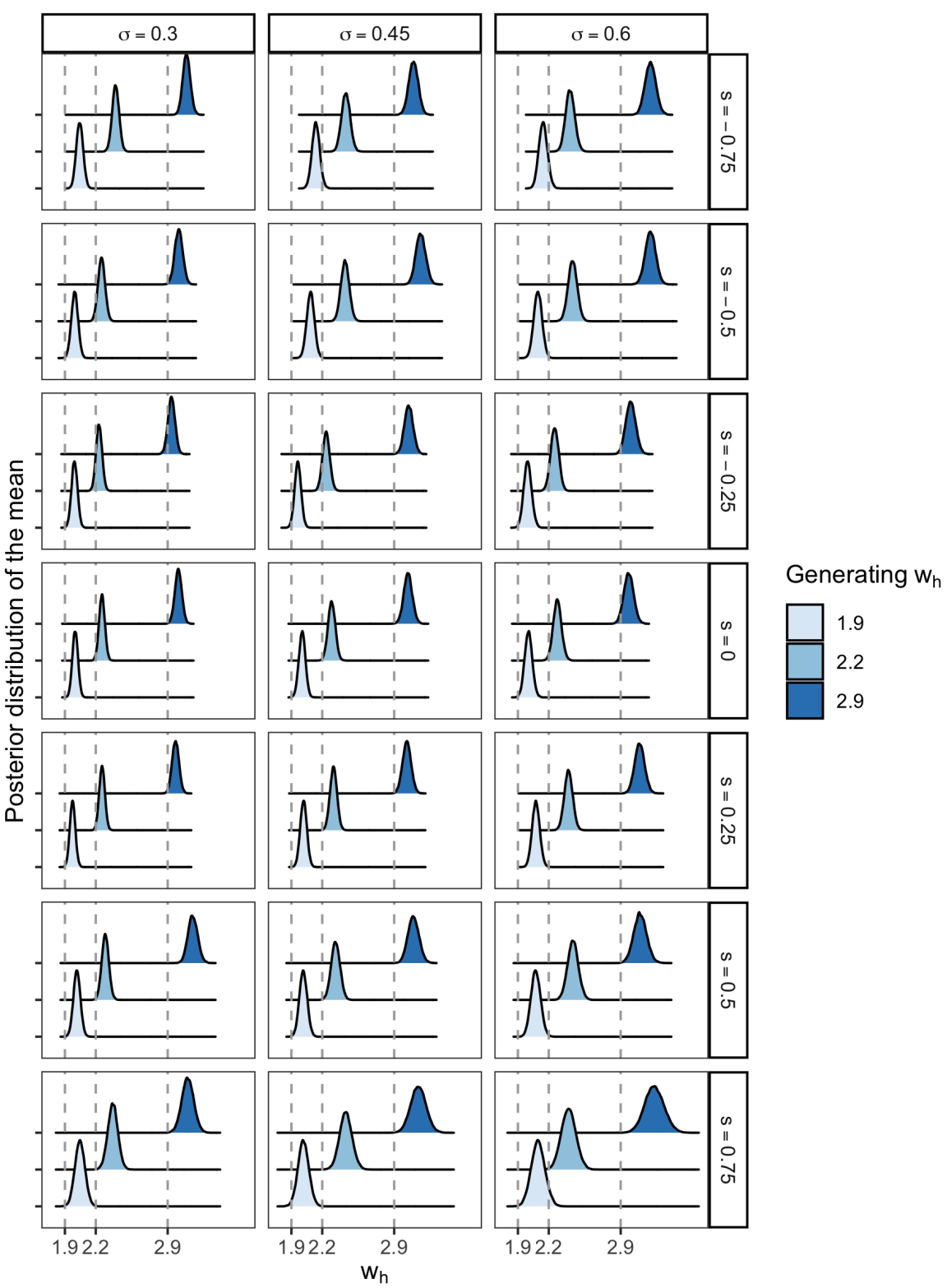

FIGURE S7 Group level posterior probability distributions of the mean $w_{h}$ parameters from the HstDDM fits of simulations varying the $\mathrm{s}$ and the $\sigma$ parameters. Each posterior distribution is based on a hierarchical Bayesian fit to 45 independent simulations. The dashed grey lines indicate the input generating parameters for the simulations which varied in the range $\{1.9,2.2,2.9\}$. Note, the $w_{t}$ parameter was always fixed to 2.2 in these simulations. 

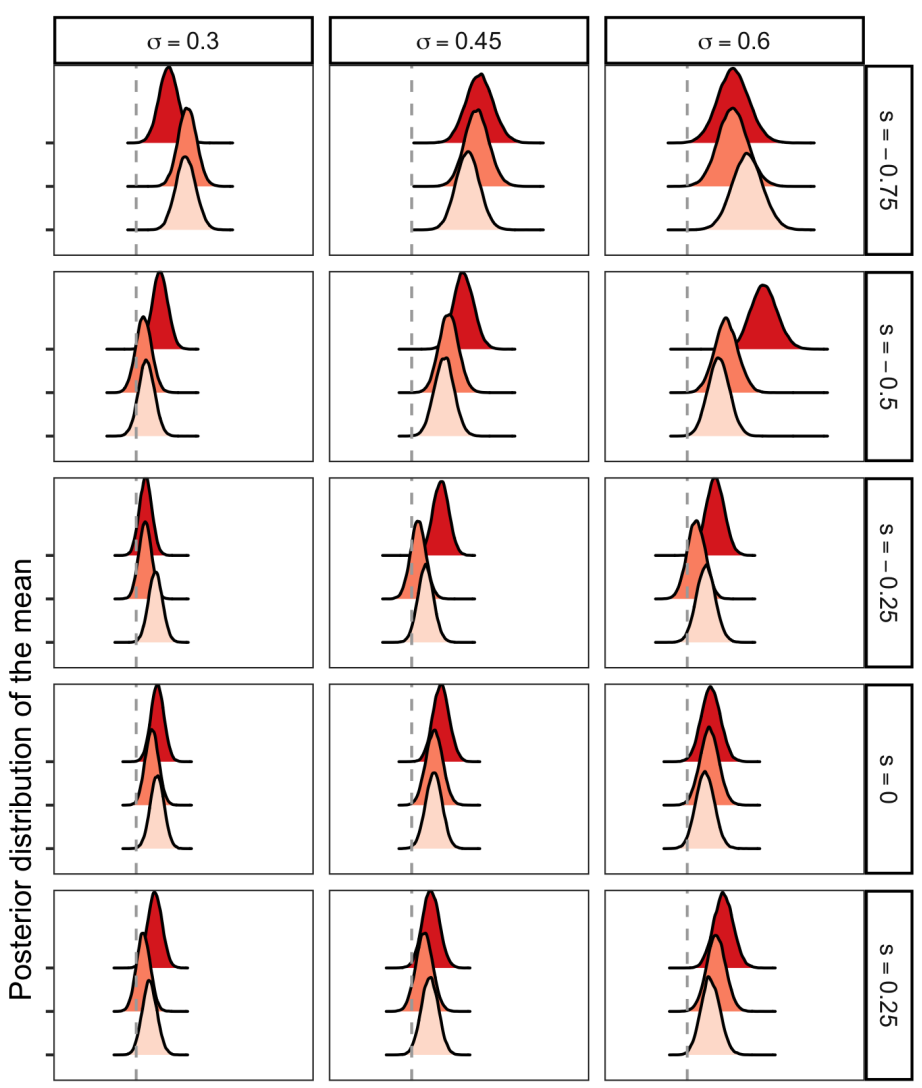

Generating $w_{h}$
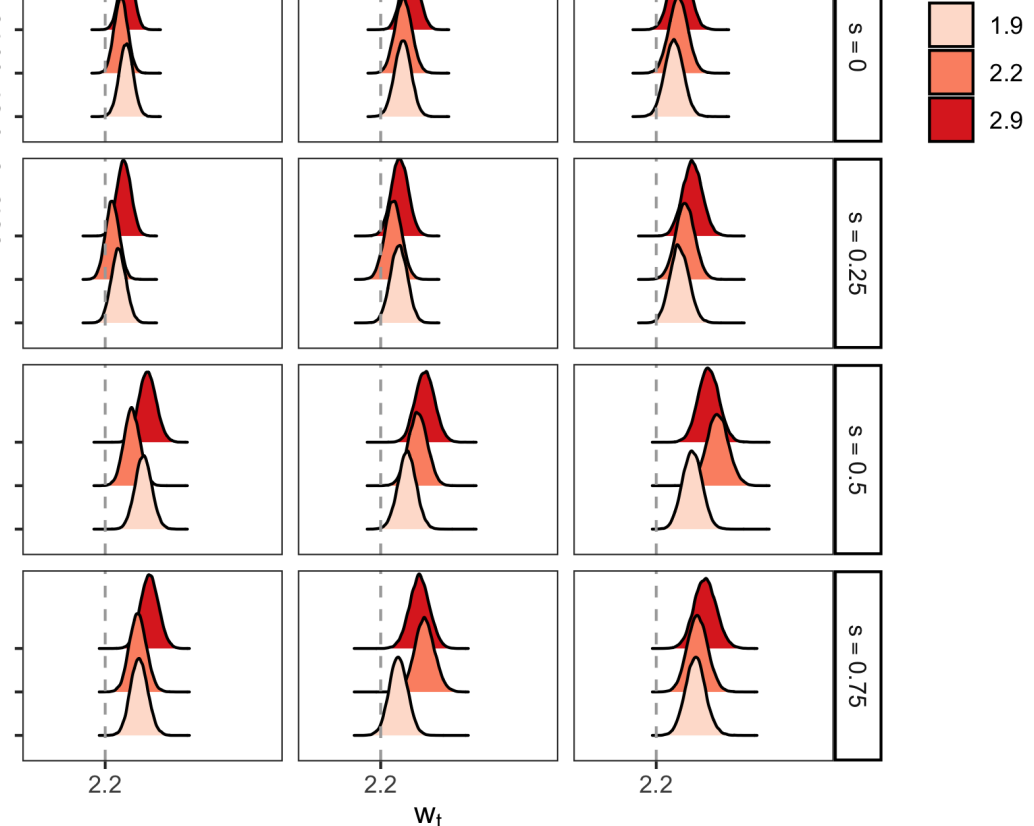

FIGURE S8 Group level posterior probability distributions of the mean $w_{t}$ parameters from the HstDDM fits of simulations varying the $\mathrm{s}$, the $\sigma$ and $w_{h}$ parameters. Each posterior distribution is based on a hierarchical Bayesian fit to 45 independent simulations. The dashed grey lines indicates the single generating parameter value of the taste weight, $w_{t}=2.2$, used for all three simulations with different health weights $\left(w_{h}\right)$. The different shading of the three distributions for $w_{t}$ in each plot refer to the corresponding generating value for the $w_{h}$ parameters $(\{1.9,2.2,2.9\})$ used in each case. The recovered value of $w_{t}$ does not differ as a function of the value of $w_{h}$ it is paired with. 

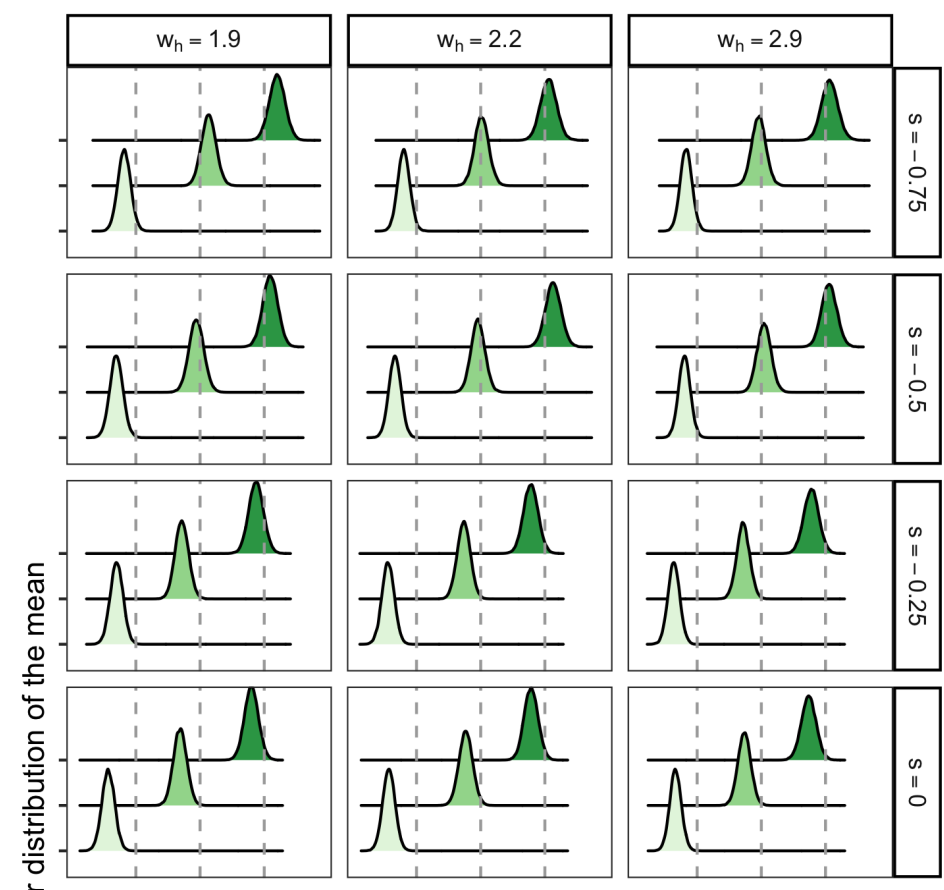

Generating $\sigma$
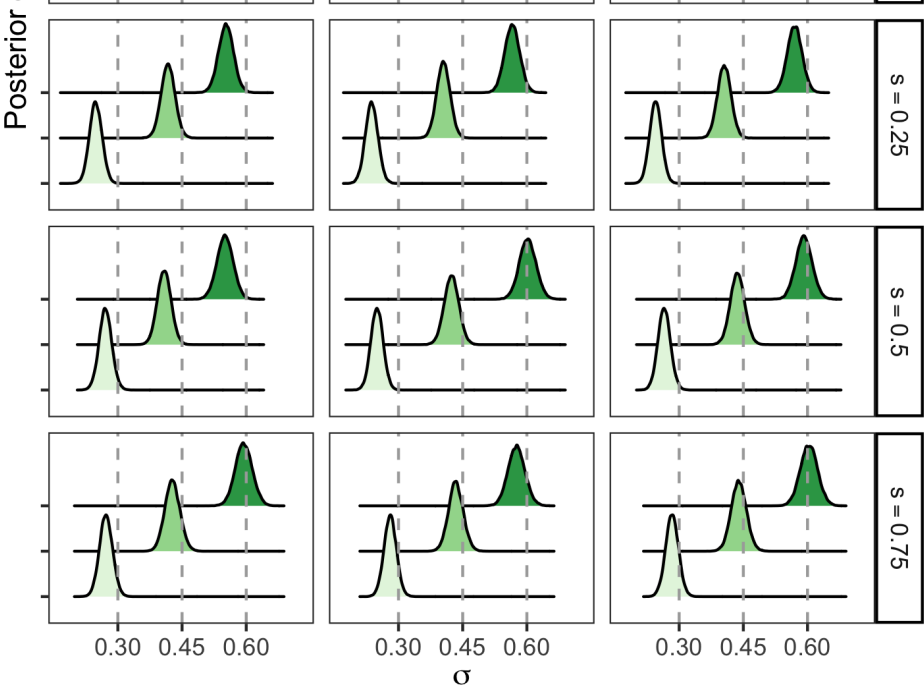

FIGURE S9 Group level posterior probability distributions of the mean $\sigma$ parameters from the HstDDM fits of simulations varying the weights and the s parameters. Each posterior distribution is based on a hierarchical Bayesian fit to 45 independent simulations. The dashed grey lines indicate the input generating parameters for the simulations. 

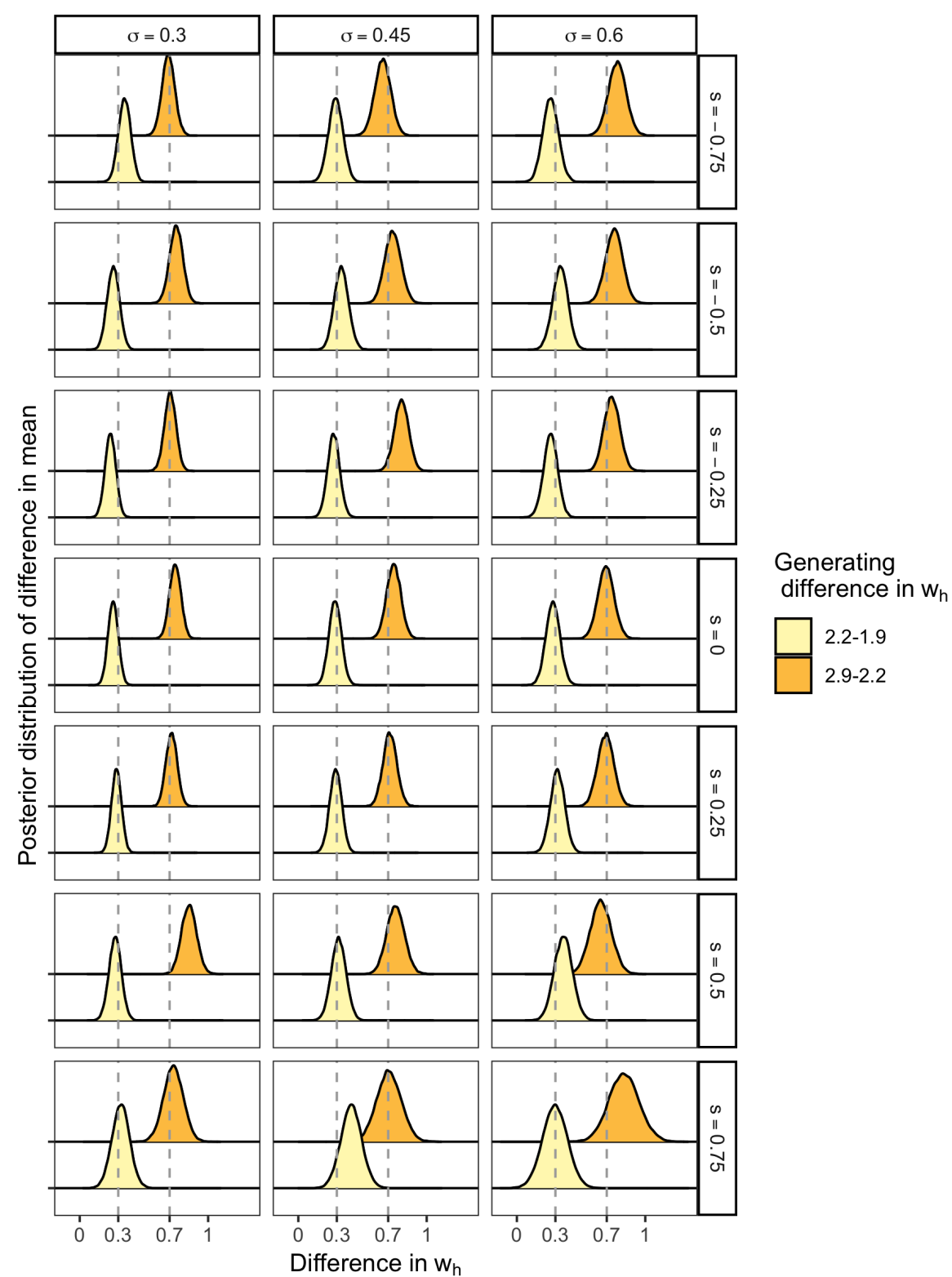

FIGURE S10 Group level posterior probability distributions of the difference in mean between $w_{h}$ parameters from HstDDM fits of separate simulations varying the $s$ and the $\sigma$ parameters. Each posterior distribution is based on a hierarchical Bayesian fit to 45 independent simulations. The dashed grey lines indicate the input generating parameter difference between simulations. 

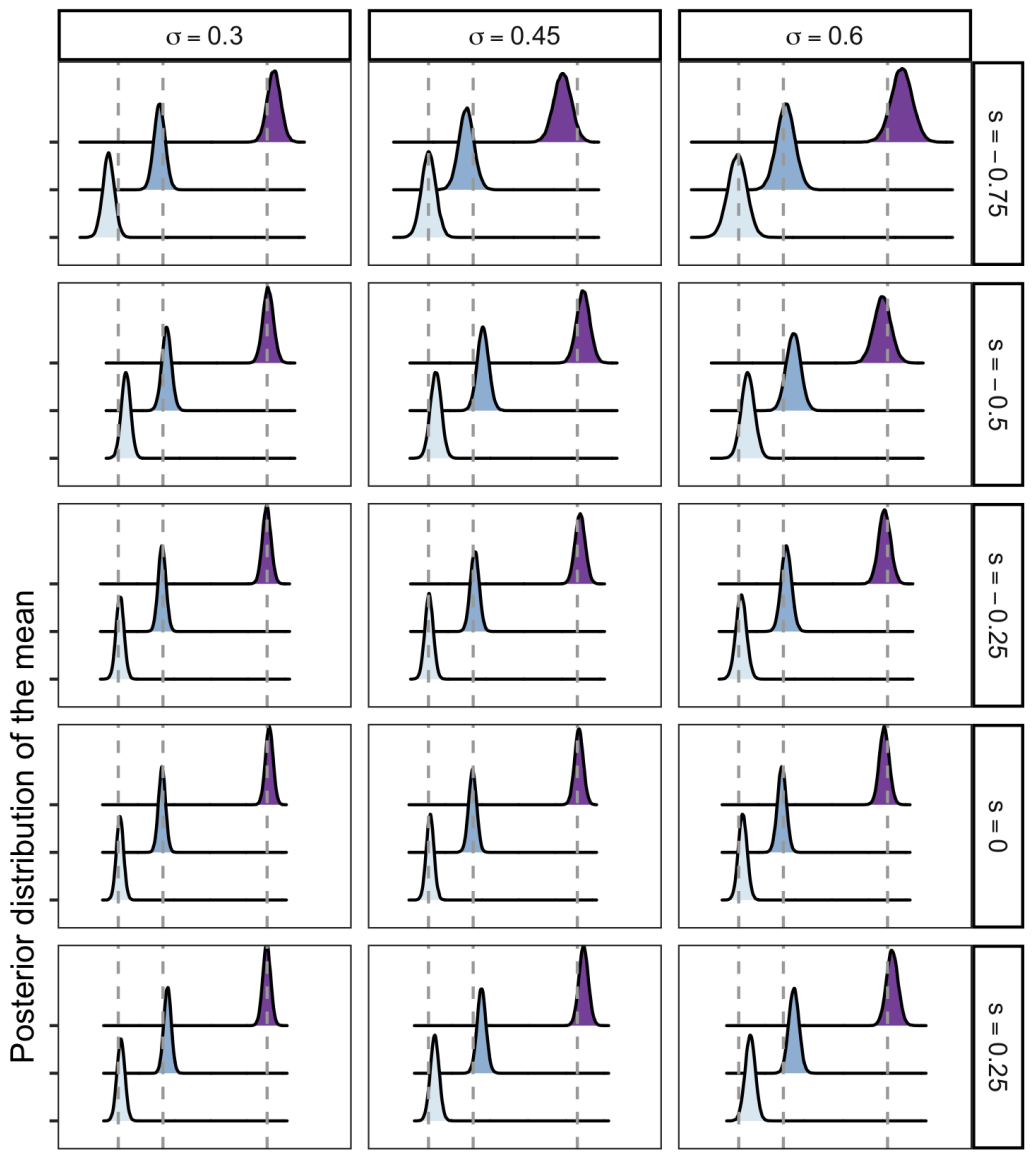

Generating
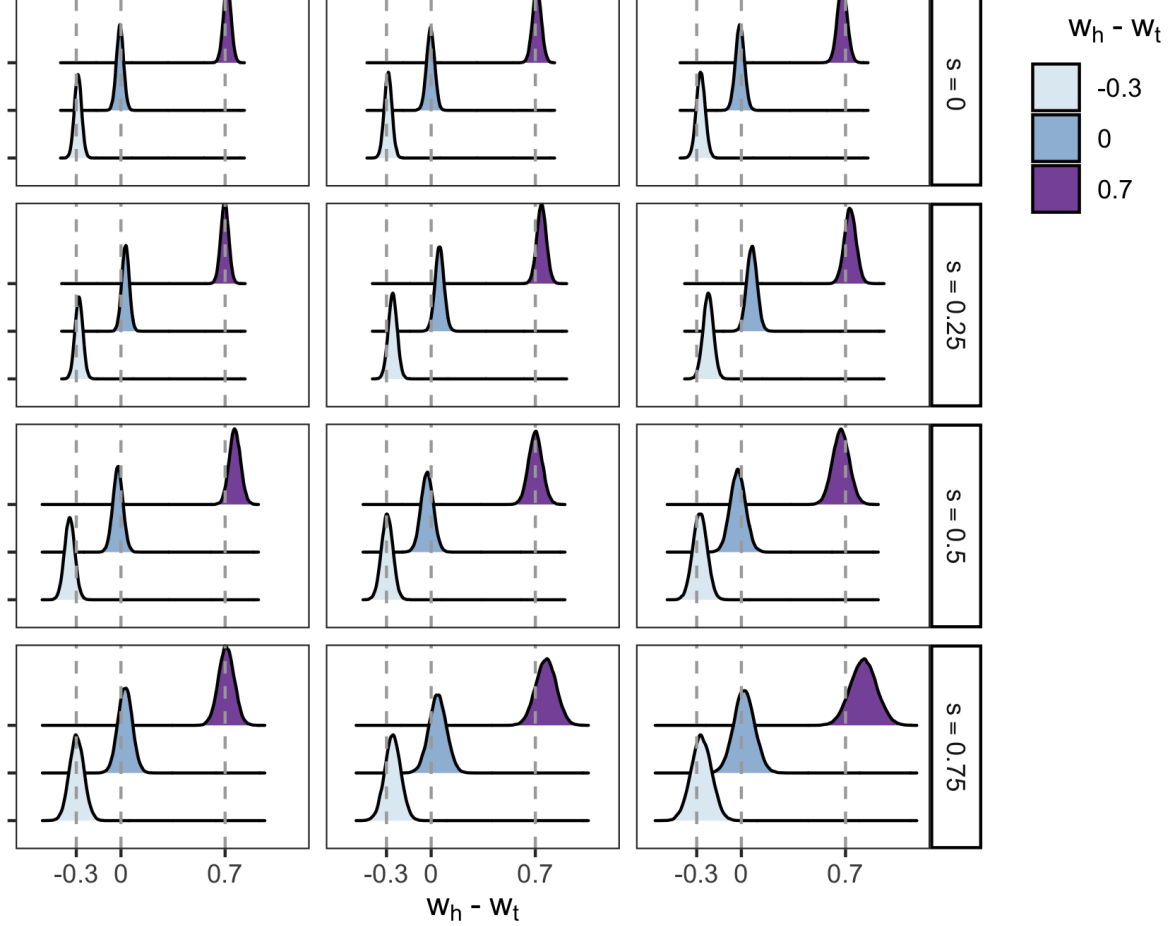

FIGURE S11 Group level posterior probability distributions of the difference in mean between $w_{h}$ and $w_{t}$ from HstDDM fits of simulations varying the $s$ and the $\sigma$ parameters. Each posterior distribution is based on a hierarchical Bayesian fit to 45 independent simulations. The dashed grey lines indicate the input generating parameter differences between the weights. 

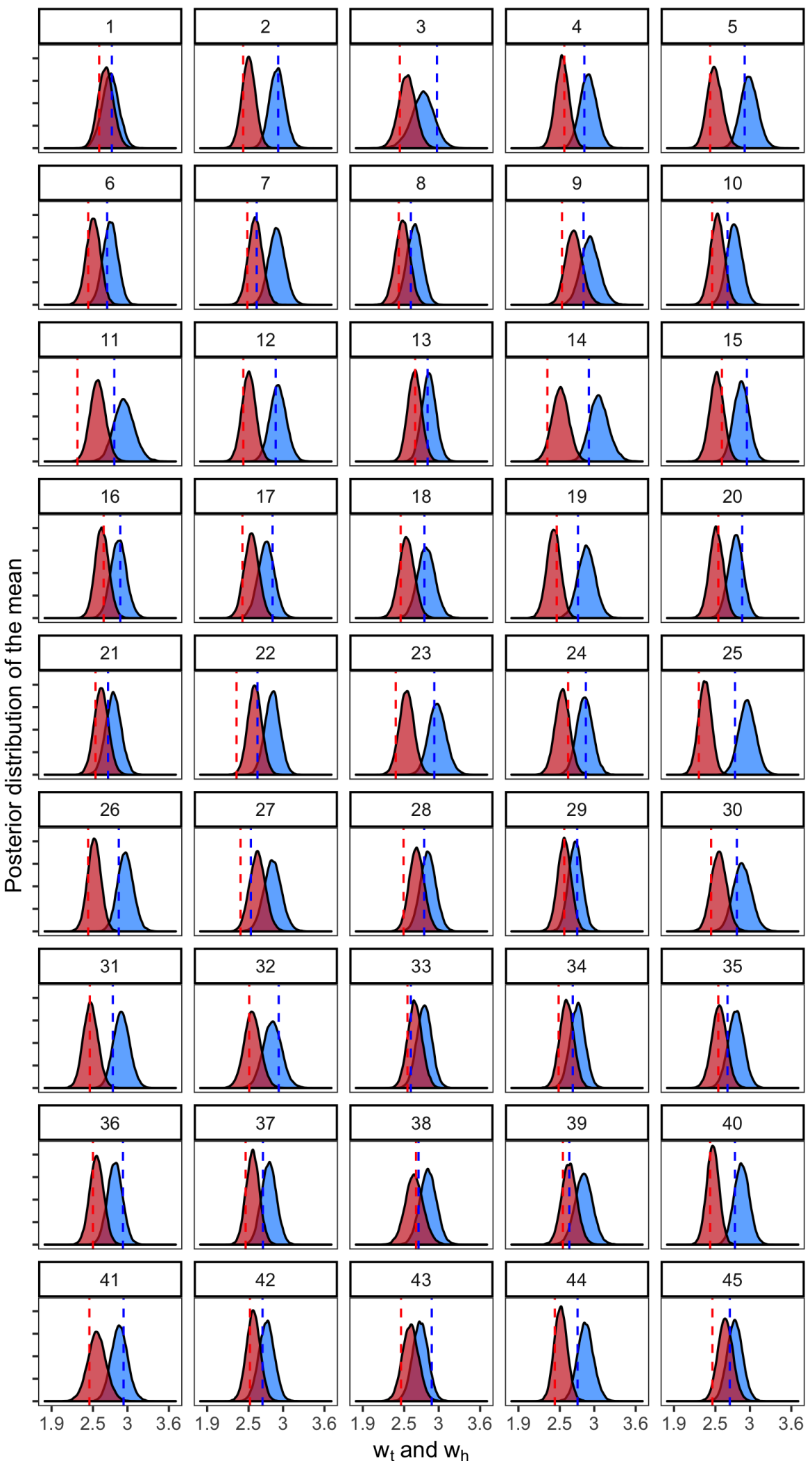

FIGURE S12 Individual level posterior probability distributions of the mean $w_{h}$,(in blue) and $w_{t}$ (in red) parameters from the HstDDM fits for each subject in the data set. The dashed lines indicate the input generating parameters used for the simulations. 

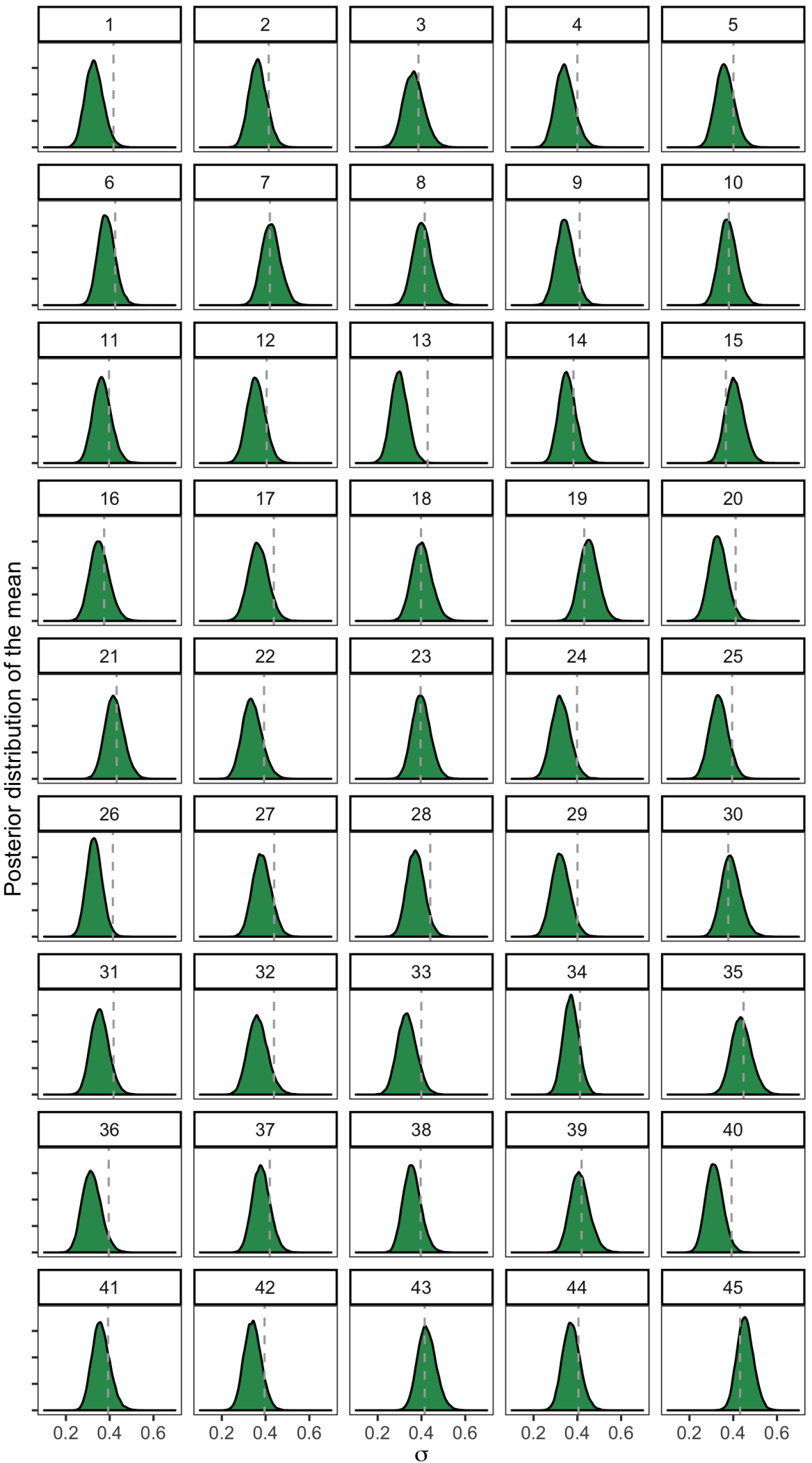

FIGURE S13 Individual level posterior probability distributions of the mean $\sigma$ parameters from the HstDDM fits for each subject in the data set. The dashed grey lines indicate the input generating parameters used for the simulations. 
Regression models of the recovered $\delta$ parameter of HaDDM

Homogeneous sets

(1)
Heterogeneous sets

(2)
Empirical fit recovery

(3)

\begin{tabular}{|c|c|c|c|}
\hline \multirow[t]{2}{*}{ Intercept } & $0.49^{* *}$ & $8.69^{* * *}$ & $0.44^{* * *}$ \\
\hline & $(0.15)$ & $(0.42)$ & $(0.07)$ \\
\hline \multirow[t]{2}{*}{ Generating $\delta$ parameter } & $1.02^{* * *}$ & $0.34^{* * *}$ & $0.54^{* * *}$ \\
\hline & $(0.002)$ & $(0.03)$ & (0.10) \\
\hline Multiple $R^{2} /$ Adjusted $R^{2}$ & 0.99 / 0.99 & $0.54 / 0.53$ & $0.51 / 0.49$ \\
\hline Number of observation $\mathrm{N}$ & 1440 & 90 & 30 \\
\hline Note: & & $.01 ;{ }^{* * *} \mathrm{p}<0$ & \\
\hline
\end{tabular}

TAB LE S1 Linear regression models explaining the estimated drift scaling parameters $\delta$ of the HaDDM as a function of the real generating parameters plus a constant intercept for the three different types of recovery fitting. Each observation in the regression models is a unique simulation. In the homogeneous sets regression, we analyzed the results of 48 hierarchical Bayesian fits to sets of 30 simulated agents. Within each set, all agents used the same aDDM parameters, but had unique fixation patterns and subjective values for each trial. The aDDM parameter combinations were different for each of the 48 sets and fits. In the heterogeneous sets regression, we analyzed the results of 3 hierarchical Bayesian fits to sets of 30 simulated agents that each had different aDDM parameters, as well as unique fixation patterns and subjective values for each trial. The empirical recovery tests used the best-fitting parameters, observed fixations, and trials for each of the 30 human participants. The significant coefficient for the generating $\delta$ parameter quantifies the association between the known generating and model estimated parameters. The significant positive intercept coefficients indicate a bias to overestimate the drift scaling parameter, $\delta$. Critically, this bias is constant, and thus, we can obtain highly accurate and unbiased estimates of parameter differences across individuals or conditions (see Table S3) 
Regression models of the recovered $w_{h}$ parameter of HstDDM

Homogeneous recovery

(1)
Heterogeneous recovery

(2)
Empirical fit recovery

(3)

$\begin{array}{lccc}\text { Intercept } & 0.02 & 0.68^{* * *} & 0.40^{* * *} \\ & (0.02) & (0.08) & (0.07) \\ \text { Generating } w_{h} \text { parameter } & 1.05^{* * *} & 0.80^{* * *} & 0.66^{* * *} \\ & (0.002) & (0.03) & (0.07)\end{array}$

Multiple $R^{2} /$ Adjusted $R^{2}$

$0.96 / 0.96$

1215
$0.77 / 0.77$

225
$0.65 / 0.64$

45

Note:

$$
{ }^{*} \mathrm{p}<0.05 ;{ }^{* *} \mathrm{p}<0.01 ;{ }^{* * *} \mathrm{p}<0.001
$$

TAB LE S 2 Linear regression models explaining the estimated drift scaling parameters $w_{h}$ of the HstDDM as a function of the real generating parameters plus a constant intercept for the three different types of recovery fitting. Each observation in the regression models is a unique simulation. In the homogeneous sets regression, we analyzed the results of 27 hierarchical Bayesian fits to sets of 45 simulated agents. Within each set, all agents used the same stDDM parameters, but had unique subjective values. The stDDM parameter combinations were different for each of the 27 sets and fits. In the heterogeneous sets regression, we analyzed the results of 5 hierarchical Bayesian fits to sets of 45 simulated agents that each had different stDDM parameters, as well as unique subjective values. The empirical recovery tests used the best-fitting parameters, observed fixations, and trials for each of the 45 human participants. The significant coefficient for the generating $w_{h}$ parameter quantifies the association between the known generating and model estimated parameters. The significant positive intercept coefficients indicate a bias to overestimate the drift scaling parameter, $w_{h}$. Critically, this bias is constant, and thus, we can obtain highly accurate and unbiased estimates of parameter differences across individuals or conditions (see Table S3) 
Regression models of the recovered difference in drift scaling parameter

Difference in $\delta s$ HaDDM

(1)

\section{Difference in $w_{h}$ HstDDM}

(2)

$\begin{array}{lcc}\text { Intercept } & 0.00 & -0.02 \\ & (0.00) & (0.01) \\ \text { Generating difference in parameters } & 1.01^{* * *} & 1.08^{* * *} \\ & (0.001) & (0.02)\end{array}$

Multiple $R^{2} /$ Adjusted $R^{2}$

$0.93 / 0.93$

$0.89 / 0.89$

Number of observation $\mathrm{N}$

1080

405

Note:

${ }^{*} \mathrm{p}<0.05 ;{ }^{* *} \mathrm{p}<0.01 ;{ }^{* * *} \mathrm{p}<0.001$

TAB LE S3 Linear regression models explaining the estimated difference in drift scaling parameters (1) $\delta$ of the HaDDM and (2) $w_{h}$ of the HstDDM as a function of the real generating parameters plus a constant intercept in the homogeneous sets recoveries. Each observation in the regression models is the difference between two unique simulations. For both the HaDDM and HstDDM, the intercept coefficient does not differ from zero indicating that the recovery of differences in drift scaling parameters is unbiased. 
Regression models of the recovered $\delta$ of the HDDM

Recovered $\delta$ parameter in the HDDM

Intercept

Generating in $\delta$ parameter

Simulate with SDE

Simulate with SDE $\times$ Generating $\delta$ parameter
$0.63^{* * *}$

(0.05)

$-0.27^{* * *}$

$-0.01$

(0.01)
$0.99 / 0.99$
Multiple $R^{2} /$ Adjusted $R^{2}$
1230

Note:

${ }^{*} \mathrm{p}<0.05 ;{ }^{* *} \mathrm{p}<0.01 ;{ }^{* * *} \mathrm{p}<0.001$

TAB LE S4 Linear regression model explaining the drift scaling parameters $\delta$ of the HDDM as a function of the real generating parameter for data simulated with the discrete bounded accumulation series (baseline for dummy Simulate with SDE $=0$ ) and the time-continuous stochastic differential equation (SDE) form of the standard drift diffusion model (dummy Simulate with SDE =1). Each observation in the regression models is a unique simulation. We analyzed the results of 41 hierarchical Bayesian fits to sets of 30 simulated agents. Within each set, all agents used the same DDM parameters, but had unique subjective values. The DDM parameter combinations were different for each of the 41 sets and fits. The linear relationship between the generating and recovered $\delta$ parameter does not differ across simulation types. However, the overestimation bias is significantly reduced for data simulated with the time-continuous SDE formula relative to the discrete bounded accumulation series. Thus, the fact that we have to use the discrete bounded accumulation series to simulate the aDDM and stDDM is one reason for the overestimation of the drift scaling parameters in those models. 